July, 1994

\title{
On the Classification of Diagonal Coset Modular Invariants
}

\author{
Terry Gannon* \\ Institut des Hautes Etudes Scientifiques, \\ 91440 Bures-sur-Yvette, France \\ Mark A. Walton ${ }^{\dagger}$ \\ Physics Department, University of Lethbridge, \\ Lethbridge, Alberta, T1K 3M4, Canada
}

\begin{abstract}
We relate in a novel way the modular matrices of GKO diagonal cosets without fixed points to those of WZNW tensor products. Using this we classify all modular invariant partition functions of $s u(3)_{k} \oplus s u(3)_{1} / s u(3)_{k+1}$ for all positive integer level $k$, and $s u(2)_{k} \oplus$ $s u(2)_{\ell} / s u(2)_{k+\ell}$ for all $k$ and infinitely many $\ell$ (in fact, for each $k$ a positive density of $\ell$ ). Of all these classifications, only that for $s u(2)_{k} \oplus s u(2)_{1} / s u(2)_{k+1}$ had been known. Our lists include many new invariants.
\end{abstract}

\footnotetext{
* e-mail: gannon@ihes.fr

$\dagger$ Supported in part by NSERC. e-mail: walton@hg.uleth.ca
} 


\section{Introduction}

It is believed that a large subset of all rational conformal field theories can be generated from the Goddard-Kent-Olive (GKO) coset construction [11]. In the prototypical example, the minimal unitary series can be identified with the cosets $s u(2)_{k} \oplus s u(2)_{1} / s u(2)_{k+1}$.

This paper is concerned with the classification of modular invariant partition functions for the diagonal GKO coset theories $g_{k} \oplus g_{\ell} / g_{k+\ell}$, where $g_{k}$ is an untwisted affine algebra, at positive integer level $k$, with horizontal subalgebra $g$. We classify what are known as physical invariants: those modular invariants with non-negative integer multiplicities, and a unique vacuum; no further conditions are imposed. The connection between this problem and the WZNW one of finding partition functions for $g_{k} \oplus g_{\ell} \oplus g_{k+\ell}^{c}$ (where $g_{k+\ell}^{c}$ is the dual of $g_{k+\ell}$ ) is well known, as is the method of constructing some of the partition functions for the coset by tensoring together partition functions for $g_{k}, g_{\ell}$, and $g_{k+\ell}$. But by means of a simple trick the coset classification is shown in Sect.2 to be equivalent to a small subset of the classification for $g_{k} \oplus g_{\ell} \oplus g_{k+\ell}$ (this is more convenient to work with than $g_{k} \oplus g_{\ell} \oplus g_{k+\ell}^{c}$ - e.g. for finding exceptionals), that can be very easily identified (see eq. (2.8c) below). In Sect. 3 we apply this to classify the coset physical invariants for certain levels $k, \ell$ and $g=s u(2)-$ half of these partition functions are not listed in e.g. [21,4]. Finally, in Sects.4 and 5 we classify the $s u(3)_{k} \oplus s u(3)_{1} / s u(3)_{k+1}$ coset theories, and find several not included in e.g. $[1,5]$.

The classification proofs follow a general strategy developed by one of us (T.G.) in several studies $[6,7,8,10]$. Modular invariants of rational conformal field theories are of two types: (i) automorphism invariants of the unextended chiral algebra, and (ii) those with non-identity fields coupling to the identity [6] (these can be interpreted [20] as invariants involving extensions of the original chiral algebra). The first are shown (in the cases under consideration) to be "locally outer automorphism" invariants. That is, all fields are composed of holomorphic and anti-holomorphic parts related by an outer automorphism (conjugation and/or simple current) of the unextended chiral algebra. The invariants of type (i) are then shown to be of a simple "global" form, and all possibilities are found.

The first step in classifying the type (ii) invariants is to find all fields that can couple to the identity field, the so-called " $\rho$-couplings". These possibilities are severely limited by $T$-invariance and the parity rule of $[6,22]$, and correspond to the possible extensions of the chiral algebra. The well-known simple current extensions are always included, and the automorphism invariants of these must be calculated. But other extensions of the chiral algebra can exist, and when they do, their automorphism invariants must all be found.

We include one table, which gives the exhaustive list of all physical invariants for $s u(3)_{k} \oplus s u(3)_{1} / s u(3)_{k+1}, \forall k$. All previously published lists seem to miss several of these, though from a "modern" perspective none of these invariants should be surprising. That our table is complete is proven in Thm.2.

\section{Cosets and WZNW tensor products}

The point of this section is to make precise the connection between the coset theories $g_{k} \oplus g_{\ell} / g_{k+\ell}$, without fixed points, and the WZNW tensor product theories $g_{k} \oplus g_{\ell} \oplus g_{k+\ell}$. 
For $\ell=1$, we show how one can restrict attention to $g_{k} \oplus g_{k+1}$. We will limit this discussion to $g=s u(n)$, but similar comments should apply to all algebras.

\subsection{Field identification}

Let $P_{++}(g, k)$ be the fundamental alcove of positive highest weights of $g_{k}$. We will write $k^{\prime}$ for the height $k+n$. We will be interested only in the 'horizontal' parts of these weights, $\lambda \in P(g)$, the weight lattice of $g$. Let $\beta_{i}, i=0, \ldots, n-1$, be the usual fundamental weights (so $\beta_{1}, \ldots, \beta_{n-1}$ span $P(g)$ ). We will identify a weight $\lambda=\sum_{a} \lambda_{a} \beta_{a}$ with its Dynkin labels $\lambda_{a}$. So $\lambda \in P_{++}(g, k)$ iff each $\lambda_{a}>0$, and $\sum_{a=1}^{n-1} \lambda_{a}<k^{\prime}$. Write $\rho=(1, \ldots, 1)$.

First define the following quantity, called $n$-ality [17]:

$$
t_{n}(\lambda) \equiv \sum_{a=1}^{n-1} a \lambda_{a}=n \lambda \cdot \beta_{n-1}(\bmod n)
$$

Then $t_{n}(\lambda) \equiv 0$ iff $\lambda \in P(g)^{*}$, the root lattice of $g . t_{n}(\lambda)$ is meant to generalize the triality of $s u(3)$.

Let $A_{k}$ denote the level $k$ simple current [12] =outer automorphism [3] which operates on $P_{++}(g, k)$ by the formula $A_{k} \lambda=\left(k^{\prime}-\sum_{a=1}^{n-1} \lambda_{a}\right) \beta_{1}+\sum_{a=2}^{n-1} \lambda_{a-1} \beta_{a}$. Usually there will be no ambiguity and we can drop the subscript $k$ on $A_{k}$.

Choose any $\lambda, \mu \in P_{++}(g, k)$, and let $S^{(k)}$ denote the modular $S$-matrix of $g_{k}$. Then:

$$
\begin{aligned}
t_{n}\left(A^{a} \lambda\right) & \equiv k a+t_{n}(\lambda)(\bmod n) \\
\left(A^{a} \lambda\right)^{2} & \equiv \lambda^{2}-2 k^{\prime} a t_{n}(\lambda) / n+k^{\prime 2} a(n-a) / n\left(\bmod 2 k^{\prime}\right) \\
S_{A^{a} \lambda, A^{b} \mu}^{(k)} & =\exp \left[2 \pi i\left(b t_{n}(\lambda)+a t_{n}(\mu)-(a+b) t_{n}(\rho)+k a b\right) / n\right] S_{\lambda \mu}^{(k)} .
\end{aligned}
$$

Define

$$
\begin{array}{r}
P_{k \ell}=\left\{(\lambda, \mu, \nu) \in P_{++}(g, k) \times P_{++}(g, \ell) \times P_{++}(g, k+\ell) \mid\right. \\
\left.t_{n}(\lambda)+t_{n}(\mu) \equiv t_{n}(\nu)+t_{n}(\rho)(\bmod n)\right\} .
\end{array}
$$

For any $(\lambda, \mu, \nu) \in P_{k \ell}$, define $A^{a}(\lambda, \mu, \nu)=\left(A_{k}^{a} \lambda, A_{\ell}^{a} \mu, A_{k+\ell}^{a} \nu\right)$. We see from $(2.2 a)$ that $(\lambda, \mu, \nu) \in P_{k \ell}$ iff $A(\lambda, \mu, \nu) \in P_{k \ell}$. This means $P_{k \ell}$ is the disjoint union of $A$-orbits. Let $P_{k \ell}^{A}$ denote $P_{k \ell}$ after modding out by $A$, i.e. it contains one and only one triple $(\lambda, \mu, \nu)$ from each $A$-orbit $\left\{A^{a}(\lambda, \mu, \nu)\right\} \subset P_{k \ell}$.

The characters of the coset theories are essentially the branching functions $b_{\lambda \nu}^{\mu}[13]$ (notice, however, we use positive weights to label the $g_{k}$ representations, rather than nonnegative ones). We know (e.g. $[5,13])$ that $b_{\lambda \nu}^{\mu} \neq 0$ iff $(\lambda, \mu, \nu) \in P_{k \ell}$, and for $(\lambda, \mu, \nu) \in$ $P_{k \ell}, b_{\lambda \nu}^{\mu}=b_{\lambda^{\prime} \nu^{\prime}}^{\mu^{\prime}}$ iff $\left(\lambda^{\prime}, \mu^{\prime}, \nu^{\prime}\right)=A^{a}(\lambda, \mu, \nu)$ for some $a$.

$(\lambda, \mu, \nu) \in P_{k \ell}$ is called a fixed point if $A^{m}(\lambda, \mu, \nu)=(\lambda, \mu, \nu)$, for some $0<m<n$. Fixed points occur iff the greatest common divisor $\operatorname{gcd}(n, k, \ell)>1$. So for $n$ prime, there will be no fixed points unless $k \equiv \ell \equiv 0(\bmod n)$. Fixed points present certain 
complications, and we will restrict attention in this paper to the simplest case, where there are no fixed points $(e . g . \ell=1)$.

Let $G$ denote the simply-connected Lie group with Lie algebra $g$. Since the centre of $G$ is diagonally embedded in the centre of $G \otimes G$, there are field identifications $[20,18,23]$. That is, we must identify different triples $(\lambda, \mu, \nu) \in P_{k \ell}$. For $g=s u(n)$ the identifications are quite simple (at least for unitary $\operatorname{cosets}^{1}$, when no fixed points are present). The characters of the coset model can be taken to be the branching functions, and $(\lambda, \mu, \nu)$ is identified with $A(\lambda, \mu, \nu)$, so that the characters are in one-to-one correspondence with the elements of $P_{k \ell}^{A}$.

\subsection{The correspondence $g_{k} \oplus g_{\ell} / g_{k+\ell} \leftrightarrow g_{k} \oplus g_{\ell} \oplus g_{k+\ell}$}

Let $\chi_{\lambda}^{(k)}$ denote the $g_{k}$ character with highest weight $\lambda$. As before, let its $S$ and $T$ modular matrices be denoted $S^{(k)}$ and $T^{(k)}$. For $(\lambda, \mu, \nu) \in P_{k \ell}^{A}$ define

$$
\tilde{c h}_{\lambda \mu \nu}=\sum_{a=0}^{n-1} \chi_{A^{a} \lambda}^{(k)} \chi_{A^{a} \mu}^{(\ell)} \chi_{A^{a} \nu}^{(k+\ell) *}
$$

Claim 1. The branching functions $\left\{b_{\lambda \nu}^{\mu} \mid(\lambda, \mu, \nu) \in P_{k \ell}^{A}\right\}$ transform modularly exactly like $\left\{\tilde{c h}_{\lambda \mu \nu} \mid(\lambda, \mu, \nu) \in P_{k \ell}^{A}\right\}$. In other words, their $S$ and $T$ matrices are equal.

Proof The $S$ and $T$ matrices for the branching functions are computed in [15] and given in $[5]$. We find that

$$
T_{\lambda \mu \nu, \lambda^{\prime} \mu^{\prime} \nu^{\prime}}=T_{\lambda \lambda^{\prime}}^{(k)} T_{\mu \mu^{\prime}}^{(\ell)} T_{\nu \nu^{\prime}}^{(k+\ell) *}
$$

The $S$-matrix for (2.4) is no harder to calculate; from $(2.2 c)$ we get a factor

$$
\sum_{a=0}^{n-1} \exp \left[2 \pi i a\left\{t_{n}\left(\lambda^{\prime}\right)+t_{n}\left(\mu^{\prime}\right)-t_{n}\left(\nu^{\prime}\right)-t_{n}(\rho)\right\} / n\right]
$$

which equals 0 unless $\left(\lambda^{\prime}, \mu^{\prime}, \nu^{\prime}\right) \in P_{k \ell}$, in which case it equals $n$. Thus the $S$-matrices are also equal, with entries

$$
S_{\lambda \mu \nu, \lambda^{\prime} \mu^{\prime} \nu^{\prime}}=n S_{\lambda \lambda^{\prime}}^{(k)} S_{\mu \mu^{\prime}}^{(\ell)} S_{\nu \nu^{\prime}}^{(k+\ell) *}
$$

QED nations

Let $\Omega_{k \ell}^{c o s}$ denote the coset commutant, i.e. the space of all modular invariant combi-

$$
\sum_{(\lambda \mu \nu),\left(\lambda^{\prime} \mu^{\prime} \nu^{\prime}\right) \in P_{k \ell}^{A}} M_{\lambda \mu \nu, \lambda^{\prime} \mu^{\prime} \nu^{\prime}} b_{\lambda \nu}^{\mu} b_{\lambda^{\prime} \nu^{\prime}}^{\mu^{\prime} *}
$$

Let $\mathcal{P}_{k \ell}^{c o s}$ denote the set of all coset physical invariants, i.e. all functions lying in the coset commutant with all coefficients $M_{\lambda \mu \nu, \lambda^{\prime} \mu^{\prime} \nu^{\prime}}$ non-negative integers, and with $M_{\rho \rho \rho, \rho \rho \rho}=1$.

1 For nonunitary diagonal cosets there are more field identifications, some not directly related to the centre of $G[19]$. 
Let $\Omega_{k \ell}^{w z w}$ denote the WZNW tensor product commutant, i.e. the space of all modular invariant combinations

$$
\sum_{\lambda, \mu, \nu, \lambda^{\prime}, \mu^{\prime}, \nu^{\prime}} \tilde{M}_{\lambda \mu \nu, \lambda^{\prime} \mu^{\prime} \nu^{\prime}} \chi_{\lambda}^{(k)} \chi_{\mu}^{(\ell)} \chi_{\nu}^{(k+\ell)} \chi_{\lambda^{\prime}}^{(k) *} \chi_{\mu^{\prime}}^{(\ell) *} \chi_{\nu^{\prime}}^{(k+\ell) *}
$$

the sum being over all $\lambda, \lambda^{\prime} \in P_{++}(g, k)$ etc. Let $\mathcal{P}_{k \ell}^{w z w}$ denote the set of all WZNW tensor product physical invariants, i.e. all functions lying in $\Omega_{k \ell}^{w z w}$ with all coefficients $\tilde{M}_{\lambda \mu \nu, \lambda^{\prime} \mu^{\prime} \nu^{\prime}}$ non-negative integers, and with $\tilde{M}_{\rho \rho \rho, \rho \rho \rho}=1$.

The claim allows us to define immediately a map $\mathcal{L}_{k \ell}^{c w}$ from $\Omega_{k \ell}^{c o s}$ into $\Omega_{k \ell}^{w z w}$, as follows. Given the coefficients $M_{\lambda \mu \nu, \lambda^{\prime} \mu^{\prime} \nu^{\prime}}$ of some coset modular invariant $(2.7 a)$, define $\tilde{M}_{\lambda \mu \nu, \lambda^{\prime} \mu^{\prime} \nu^{\prime}}=M_{\lambda \mu \nu^{\prime}, \lambda^{\prime} \mu^{\prime} \nu}\left(\right.$ note the $\nu, \nu^{\prime}$ switch $^{2}$ ). Then $\tilde{M}$ will define a WZNW tensor product modular invariant (2.7b). Equivalently, this amounts to replacing each $b_{\lambda \nu}^{\mu}$ with $\tilde{c h}_{\lambda \mu \nu}$. From the claim, modular invariance is assured. This map is one-to-one; it takes the coset physical invariants into the WZNW tensor product physical invariants; however it is not onto. In particular, the image of $\mathcal{L}_{k \ell}^{c w}$ consists of all modular invariants $\tilde{Z}$ in $\Omega_{k \ell}^{w z w}$ satisfying:

(i) $\tilde{M}_{\lambda \mu \nu, \lambda^{\prime} \mu^{\prime} \nu^{\prime}}=0$ unless

$$
t_{n}(\lambda)+t_{n}(\mu)-t_{n}\left(\nu^{\prime}\right) \equiv t_{n}\left(\lambda^{\prime}\right)+t_{n}\left(\mu^{\prime}\right)-t_{n}(\nu) \equiv t_{n}(\rho) \quad(\bmod n)
$$

(ii) for all $a, b=0, \ldots, n-1$,

$$
\tilde{M}_{A^{a} \lambda A^{a} \mu A^{b} \nu, A^{b} \lambda^{\prime} A^{b} \mu^{\prime} A^{a} \nu^{\prime}}=\tilde{M}_{\lambda \mu \nu, \lambda^{\prime} \mu^{\prime} \nu^{\prime}}
$$

From the equation $\tilde{M}=S^{\dagger} \tilde{M} S$, it is not difficult to show that (i) holds iff (ii) holds, iff

$$
\tilde{M}_{A_{k} \rho A_{\ell} \rho \rho, \rho \rho A_{k+\ell} \rho}=\tilde{M}_{\rho \rho A_{k+\ell} \rho, A_{k} \rho A_{\ell} \rho \rho}=\tilde{M}_{\rho \rho \rho, \rho \rho \rho}
$$

holds, for any physical invariant $\tilde{M}$. For example, that $(2.8 c)$ implies (i) follows by looking at the $\left(A_{k} \rho A_{\ell} \rho \rho, \rho \rho A_{k+\ell} \rho\right)$-entry of $\tilde{M}=S^{\dagger} \tilde{M} S$, and using (2.2c) and the fact that $S_{\rho \rho \rho, \lambda \mu \nu}>0$.

$\tilde{Z} \in \Omega_{k \ell}^{w z w}$ can be written as a sesquilinear combination of $\tilde{c h}_{\lambda \mu \nu}$ over $(\lambda, \mu, \nu) \in P_{k \ell}^{A}$, iff (2.8) is satisfied. Call this subspace $\Omega_{k \ell}^{w z w, A}$. Then $\mathcal{L}_{k \ell}^{c w}$ defines an isomorphism between $\Omega_{k \ell}^{c o s}$ and $\Omega_{k \ell}^{w z w, A}$, and a bijection between the physical invariants $\mathcal{P}_{k \ell}^{c o s}$ and $\mathcal{P}_{k \ell}^{w z w, A}=$ $\mathcal{P}_{k \ell}^{w z w} \cap \Omega_{k \ell}^{w z w, A}$.

The same "switch" of weights works in establishing a bijection between the physical invariants (and commutants) of $g \oplus h$ and $g \oplus h^{c}$, where $g, h$ are any chiral (e.g. affine) algebras and $h^{c}$ denotes the dual of $h$, corresponding to modular matrices which are complex conjugates of those of $h$. This bijection is difficult to establish by other means, since

2 Perhaps the simplest way to see the necessity of this switch is to write a nonzero element of the WZNW $T$ matrix as $\exp \left\{2 \pi i\left(\left(h_{\lambda}+h_{\mu}+h_{\nu}\right)-\left(h_{\lambda^{\prime}}+h_{\mu^{\prime}}+h_{\nu^{\prime}}\right)\right)\right\}=\exp \left\{2 \pi i\left(\left(h_{\lambda}+h_{\mu}-\right.\right.\right.$ $\left.\left.\left.h_{\nu^{\prime}}\right)-\left(h_{\lambda^{\prime}}+h_{\mu^{\prime}}-h_{\nu}\right)\right)\right\}$. Here $h_{\lambda}+h_{\mu}+h_{\nu}$ is a WZNW conformal weight, while $h_{\lambda}+h_{\mu}-h_{\nu^{\prime}}$ is a coset conformal weight $(\bmod 1)$. 
most physical invariants $M^{g h}$ of $g \oplus h$ cannot be factorized as $M^{g} \otimes M^{h}$. This switch is helpful in generating coset modular invariants which would be difficult to find otherwise, though as we saw further conditions must be satisfied in order that this correspondence be extended to coset theories.

Incidentally, it was shown in [6] that the Roberts-Terao-Warner lattice method succeeds in generating any WZNW commutant. The mapping $\mathcal{L}_{k \ell}^{c w}$ tells us then that the lattice method can be successfully extended to the diagonal cosets without fixed points, where it will also be complete.

Thus to find all physical invariants of $s u(n)_{k} \oplus s u(n)_{\ell} / s u(n)_{k+\ell}$ when $\operatorname{gcd}(n, k, \ell)=1$, it suffices to find all physical invariants of $s u(n)_{k} \oplus s u(n)_{\ell} \oplus s u(n)_{k+\ell}$ which satisfy $(2.8 c)$.

\subsection{Simplification when $\ell=1$}

When one of the levels, say $\ell$, equals 1 , the correspondence given above relating the cosets to WZNW tensor products simplifies. The reason is that $A_{1}$ acts transitively on the $n$ weights in $P_{++}(g, 1)$, so modding by $A$ essentially removes that factor.

When $\ell=1$, there can be no fixed points, regardless of the value of $k$ or $n$, so the comments in this section apply to any level $k$ and any $s u(n)$.

Define $P_{k}=\left\{(\lambda, \mu) \in P_{++}(g, k) \times P_{++}(g, k+1) \mid t_{n}(\lambda) \equiv t_{n}(\mu)(\bmod n)\right\}$. There is an obvious bijection between $P_{k}$ and $P_{k, 1}^{A}$ : identify $(\lambda, \mu)$ with the $A$-orbit containing $(\lambda, \rho, \mu)$. Each $A$-orbit in $P_{k, 1}$ contains exactly one triple of the form $(\lambda, \rho, \mu)$, so we may identify the orbit, and hence the corresponding element of $P_{k, 1}^{A}$, with such a triple.

Define $\Omega_{k}^{w z w}$ to be the WZNW commutant for the direct sum $s u(n)_{k} \oplus s u(n)_{k+1}$.

Define a map, $\mathcal{L}_{k}^{c w}$, between $\Omega_{k, 1}^{c o s}$ and $\Omega_{k}^{w z w}$ as follows: let

$$
Z=\sum_{\lambda \mu, \kappa \nu \in P_{k}} M_{\lambda \rho \mu, \kappa \rho \nu} b_{\lambda \mu}^{\rho} b_{\kappa \nu}^{\rho *}
$$

be any modular invariant in $\Omega_{k, 1}^{c o s}$, then define

$$
\mathcal{L}_{k}^{c w}(Z)=\sum_{\lambda \mu, \kappa \nu \in P_{k}} M_{\lambda \rho \mu, \kappa \rho \nu} \sum_{a=0}^{n-1} \chi_{A^{a} \lambda}^{(k)} \chi_{A^{a} \nu}^{(k+1)} \chi_{A^{a} \kappa}^{(k) *} \chi_{A^{a} \mu}^{(k+1) *}
$$

(Notice again the switch: $\mu \leftrightarrow \nu$.)

Claim 2. $\quad \mathcal{L}_{k}^{c w}(Z)$ is a modular invariant.

Proof The arguments here are similar to those used earlier in proving Claim 1, except that here we must transform the partition functions, instead of simply investigating the modular behaviour of the characters $b$ and $\tilde{c h}$ as was done there.

$T$-invariance follows as for Claim 1. $S$-invariance is also similar to Claim 1: the calculation produces the sum

$$
\sum_{a=0}^{n-1} \exp \left[2 \pi i a\left\{t_{n}(\lambda)+t_{n}(\nu)-t_{n}(\kappa)-t_{n}(\mu)\right\} / n\right]
$$


which equals 0 unless $t_{n}(\lambda)+t_{n}(\nu) \equiv t_{n}(\kappa)+t_{n}(\mu)(\bmod n)$, i.e. unless there exists an $a$ such that both $A^{a}(\lambda, \mu), A^{a}(\kappa, \nu) \in P_{k}$, in which case the sum equals $n$. QED

As before, $\mathcal{L}_{k}^{c w}$ is one-to-one. It also is not onto; its image is the subspace of $\Omega_{k}^{w z w}$ containing those modular invariants $\bar{Z}=\sum \bar{M}_{\lambda \mu, \kappa \nu} \chi_{\lambda} \chi_{\mu} \chi_{\kappa}^{*} \chi_{\nu}^{*}$ satisfying:

(a) $\bar{M}_{\lambda \mu, \kappa \nu}=0$ unless

$$
t_{n}(\lambda)+t_{n}(\mu) \equiv t_{n}(\kappa)+t_{n}(\nu) \quad(\bmod n)
$$

(b) for all $a=0,1, \ldots, n-1$,

$$
\bar{M}_{\lambda \mu, \kappa \nu}=\bar{M}_{A^{a} \lambda A^{a} \mu, A^{a} \kappa A^{a} \nu}
$$

For any physical invariant $\bar{M}$, (a) holds iff (b) does, iff

$$
\bar{M}_{\rho \rho, \rho \rho}=\bar{M}_{A \rho A \rho, A \rho A \rho}
$$

As before, this follows from looking at $\bar{M}=S^{\dagger} \bar{M} S$.

Let $\Omega_{k}^{w z w, A}$ denote this subspace; then $\mathcal{L}_{k}^{c w}$ is an isomorphism between $\Omega_{k, 1}^{c o s}$ and $\Omega_{k}^{w z w, A}$, and a bijection between $\mathcal{P}_{k, 1}^{c o s}$ and those physical invariants in $\Omega_{k}^{w z w, A}$.

Thus to find all physical invariants of $s u(n)_{k} \oplus s u(n)_{1} / s u(n)_{k+1}$, it suffices to find all physical invariants of $s u(n)_{k} \oplus s u(n)_{k+1}$ which satisfy $(2.11 c)$.

\section{An illustration: The cosets $s u(2)_{k} \oplus s u(2)_{\ell} / s u(2)_{k+\ell}$}

As a simple illustration of the results of the previous section, we will read off from known results for $s u(2)_{k} \oplus s u(2)_{k+1}$ and $s u(2)_{k} \oplus s u(2)_{\ell} \oplus s u(2)_{m}$ the complete list of $s u(2)_{k} \oplus s u(2)_{\ell} / s u(2)_{k+\ell}$ partition functions, for certain choices of $k$ and $\ell$. In Sect.3.1 we give a new argument, based on Sect.2.3 and [7], for the $\ell=1$ proof in [4]. In Sect.3.2 we write down all the "obvious" $s u(2)_{k} \oplus s u(2)_{\ell} \oplus s u(2)_{k+\ell}$ partition functions for general levels $k, \ell$, that satisfy $(2.8 \mathrm{c})$. In Sect.3.3 we prove this list is complete whenever the three greatest common divisors $\operatorname{gcd}(k+2, \ell+2), \operatorname{gcd}(k+2, \ell)$ and $\operatorname{gcd}(k, \ell+2)$ are all $\leq 3$.

The completeness proof for all $k$ and $\ell$ may now be within sight, thanks to [7] and recent work by Stanev [24]; here we only prove it for the $k, \ell$ mentioned above.

$3.1 s u(2)_{k} \oplus \operatorname{su}(2)_{1} / s u(2)_{k+1}$

The easiest cosets are of the form $s u(2)_{k} \oplus s u(2)_{1} / s u(2)_{k+1}$, and were classified in [4]. These constitute the minimal series. We will give an alternative argument here.

From Sect.2.3 we know we must find all physical invariants of $s u(2)_{k} \oplus s u(2)_{k+1}$ which satisfy eqs.(2.11). Using [7] we can easily find for example all physical invariants for $s u(2)_{k_{1}} \oplus s u(2)_{k_{2}}$, when $\operatorname{gcd}\left(k_{1}+2, k_{2}+2\right) \leq 3$. Here we have $k_{1}=k, k_{2}=k+1$, so this gcd condition is indeed satisfied. The only physical invariants turn out to be:

(1) $\mathcal{A}_{k} \otimes \mathcal{A}_{k+1}$, for all $k$;

(2) $\mathcal{A}_{k} \otimes \mathcal{D}_{k+1}$, for all odd $k$; 
(3) $\mathcal{D}_{k} \otimes \mathcal{A}_{k+1}$, for all even $k$;

(4) the exceptionals $\mathcal{A}_{9} \otimes \mathcal{E}_{10}, \mathcal{E}_{10} \otimes \mathcal{A}_{11}, \mathcal{A}_{15} \otimes \mathcal{E}_{16}, \mathcal{E}_{16} \otimes \mathcal{A}_{17}, \mathcal{A}_{27} \otimes \mathcal{E}_{28}$, and $\mathcal{E}_{28} \otimes \mathcal{A}_{29}$. The physical invariants $\mathcal{A}_{k}, \mathcal{D}_{k}$ (for $k$ even), and $\mathcal{E}_{k}$ (for $k=10,16,28$ ) are the physical invariants for $s u(2)_{k}$ (their subscript is their level); they can be found in [4].

It is straightforward to check that (2.11c) is satisfied by all the invariants in (1)-(4). Thus to each of the WZNW physical invariants given above, there is a coset physical invariant.

This example is uncharacteristically simple: for one thing, relatively few physical invariants of $s u(n)_{k} \oplus s u(n)_{k+1}$ or $s u(n)_{k} \oplus s u(n)_{\ell} \oplus s u(n)_{k+\ell}$ will be tensor products of physical invariants of $s u(n)$; for another thing, (2.8) and (2.11) will usually be violated by most physical invariants (e.g. (2.8) cannot be satisfied by any diagonal invariant).

\section{2 $s u(2)_{k} \oplus s u(2)_{\ell} \oplus s u(2)_{k+\ell}$}

Now let us write down the "obvious" invariants for $s u(2)_{k} \oplus s u(2)_{\ell} \oplus s u(2)_{k+\ell}$, for all $k, \ell$. Some of these are included in [21], but some are not. For completeness, we will include here the invariants when $k$ and $\ell$ are both even (in which case there will be fixed point), without discussing the resolution of those fixed points. We will begin by listing the "simple current invariants" [12]. If either $k$ or $\ell$ is odd, we find exactly 2 of these; if both $k$ and $\ell$ are even there are exactly 6 .

A simple current for $s u(2)_{k} \oplus s u(2)_{\ell} \oplus s u(2)_{k+\ell}$ can be written as a triple $J=$ $\left(J_{1}, J_{2}, J_{3}\right)$, each $J_{a}=0$ or 1 . It acts on a weight $\lambda=\left(\lambda_{1}, \lambda_{2}, \lambda_{3}\right)$ by

$$
(J \lambda)_{a}=\left\{\begin{array}{cc}
\lambda_{a} & \text { if } J_{a}=0 \\
k_{a}+2-\lambda_{a} & \text { if } J_{a}=1
\end{array},\right.
$$

where we define $k_{1}=k, k_{2}=\ell$ and $k_{3}=k+\ell$. Define also the quantities $J^{2}$ and $J \cdot \lambda$ by

$$
J^{2} \equiv \sum_{a=1}^{3} J_{a} k_{a}(\bmod 4), \quad J \cdot \lambda \equiv \sum_{a=1}^{3} J_{a} \lambda_{a}(\bmod 2) .
$$

A simple current invariant [12] $M$ of $s u(2)_{k} \oplus s u(2)_{\ell} \oplus s u(2)_{k+\ell}$ obeys the "local" selection rule

$$
M_{\lambda \mu} \neq 0 \Rightarrow \mu=J \lambda \text { for some } J .
$$

More precisely, any simple current $J$ with $J^{2}$ even can be used to define a simple current invariant $M(J)$, in the following way:

$$
[M(J)]_{\lambda \mu}=\sum_{m=0}^{1} \delta_{J^{m} \lambda, \mu} \delta_{2}\left[J \cdot(\lambda-\rho)+m J^{2} / 2\right],
$$

where $\delta_{n}[x]=1$ for $x \equiv 0(\bmod n)$, and vanishes otherwise. The only other simple current invariant we will need we will call $M^{110}$; it is an invariant iff $k \equiv \ell \equiv 0(\bmod 4)$, and is defined by

$$
\left(M^{110}\right)_{\lambda \mu}=\left\{\begin{array}{lll}
1 & \text { if } \mu=J^{\prime} \lambda, & \text { where } J^{\prime} \equiv\left(\lambda_{2}+1, \lambda_{1}+1,0\right)(\bmod 2) \\
0 & \text { otherwise }
\end{array} .\right.
$$


All simple current invariants were explicitly found for all levels and arbitrary numbers of $s u(2)$ factors, in [7]. In our case, there will be either 30 or 6 of them, depending on whether or not both $k$ and $\ell$ are even. We are only interested here in those which also satisfy $(2.8 c)$. The complete list of solutions is:

(sc.1) $k, \ell$ both odd: $M(111)$ and $M(111) M(001)$;

(sc.2) $k$ odd, $\ell$ even: $M(111)$ and $M(111) M(010)$;

(sc.3) $k \equiv \ell \equiv 0(\bmod 4): \quad M(111), M(111) M^{110}, M(111) M(100), M(111) M(010)$, $M(111) M(001)$, and $M(100) M(010) M(001)$;

(sc.4) $k$ even, $\ell \equiv 2(\bmod 4): \quad M(111), M(111) M(100), M(111) M(010), M(111) M(001)$, $M(111) M(100) M(010)$ and $M(111) M(010) M(001)$.

In the special case where $k=\ell$, we may take the conjugations $M^{c}$ of each of these, defined by

$$
\left(M^{c}\right)_{\lambda \mu}=M_{\lambda, \mu_{2} \mu_{1} \mu_{3}} .
$$

$M$ will obey $(2.8 c)$ iff $M^{c}$ will.

It is curious that the numbers of invariants in (sc) are precisely the numbers of (unconstrained) simple current invariants for $s u(2)_{k} \oplus s u(2)_{\ell}$.

The importance of simple current invariants (and their conjugations) is that in all cases we know, they represent "almost all" of the physical invariants. The remaining invariants are called the exceptionals; most of these can be built up from the $\mathcal{E}_{10}, \mathcal{E}_{16}$ and $\mathcal{E}_{28}$ exceptionals of the $s u(2)_{k}$ classification [4]. Those of this form which satisfy (2.8c) are:

(e.1) $m=10, n$ odd: $\quad\left(\mathcal{A}_{n} \otimes \mathcal{E}_{10} \otimes \mathcal{A}_{n+10}\right) M(111)$ and $\left(\mathcal{A}_{n} \otimes \mathcal{A}_{10-n} \otimes \mathcal{E}_{10}\right) M(111)$;

(e.2) $m=10, n$ even: $\quad\left(\mathcal{E}_{10} \otimes \mathcal{A}_{n} \otimes \mathcal{A}_{n+10}\right) M(111),\left(\mathcal{E}_{10} \otimes \mathcal{D}_{n} \otimes \mathcal{A}_{n+10}\right) M(111),\left(\mathcal{E}_{10} \otimes\right.$ $\left.\mathcal{A}_{n} \otimes \mathcal{D}_{n+10}\right) M(111),\left(\mathcal{E}_{10} \otimes \mathcal{D}_{n} \otimes \mathcal{D}_{n+10}\right) M(111)$ and the four additional invariants obtained by interchanging the first and third components here;

(e.3) $m=16$ or $28, n$ odd: $\quad\left(\mathcal{A}_{n} \otimes \mathcal{E}_{m} \otimes \mathcal{A}_{m+n}\right) M(101)$ and $\left(\mathcal{A}_{n} \otimes \mathcal{A}_{m-n} \otimes \mathcal{E}_{m}\right) M(110)$; (e.4) $m=16$ or $28, n$ even: $\quad\left(\mathcal{E}_{m} \otimes \mathcal{A}_{n} \otimes \mathcal{A}_{m+n}\right) M(011),\left(\mathcal{E}_{m} \otimes \mathcal{D}_{n} \otimes \mathcal{A}_{m+n}\right) M(011)$, $\left(\mathcal{A}_{n} \otimes \mathcal{A}_{m-n} \otimes \mathcal{E}_{m}\right) M(110)$, and $\left(\mathcal{D}_{n} \otimes \mathcal{A}_{m-n} \otimes \mathcal{E}_{m}\right) M(110) ;$

(e.5) $m, n \in\{10,16,28\}$ (say $m \leq n)$ : the additional invariants in this case are $\left(\mathcal{E}_{m} \otimes \mathcal{E}_{n} \otimes\right.$ $\left.\mathcal{A}_{m+n}\right) M(111),\left(\mathcal{E}_{10} \otimes \mathcal{E}_{10} \otimes \mathcal{D}_{20}\right) M(111)$ and (if $\left.m<n\right)\left(\mathcal{E}_{m} \otimes \mathcal{A}_{n-m} \otimes \mathcal{E}_{n}\right) M(111)$.

Of course the first and second components can be interchanged, and the conjugation (3.4) can be taken if $k=\ell$. The lists (sc) and (e) will almost certainly exhaust all but finitely many of the physical invariants for $s u(2)_{k} \oplus s u(2)_{\ell} \oplus s u(2)_{k+\ell}$ satisfying (2.8c).

The exceptionals for $s u(2)_{k_{1}} \oplus s u(2)_{k_{2}}$ not built from the $\mathcal{E}_{m}$ occur at $\left(k_{1}, k_{2}\right)=(4,4)$, $(6,6),(8,8),(10,10),(2,10),(3,8),(3,28)$, and $(8,28)$ (see Ref. [9]). Call these $\mathcal{E}_{4,4}$, etc. These can be used to construct further invariants for us:

(sp.1) $k=\ell=4: \quad \mathcal{E}_{4,4} \otimes \mathcal{D}_{8}$;

(sp.2) $k=\ell=6: \quad \mathcal{E}_{6,6} \otimes \mathcal{D}_{12}$;

(sp.3) $k=\ell=8: \quad \mathcal{E}_{8,8} \otimes \mathcal{D}_{16}$, and $\mathcal{E}_{8,8} \otimes \mathcal{E}_{16} ;$

(sp.4) $k=\ell=10: \quad \mathcal{E}_{10,10} \otimes \mathcal{D}_{20}$;

(sp.5) $k=2, \ell=10: \quad \mathcal{E}_{2,10} \otimes \mathcal{D}_{12}$;

(sp.6) $k=2, \ell=8: \quad \mathcal{E}_{2,10} \otimes \mathcal{D}_{8}$ (interchange second and third components); 
(sp.7) $k=3, \ell=8: \quad\left(\mathcal{E}_{3,8} \otimes \mathcal{A}_{11}\right) M(101)$;

(sp.8) $k=3, \ell=5: \quad\left(\mathcal{E}_{3,8} \otimes \mathcal{A}_{5}\right) M(101)$ (interchange second and third components);

(sp.9) $k=3, \ell=28: \quad\left(\mathcal{E}_{3,28} \otimes \mathcal{A}_{31}\right) M(101)$;

(sp.10) $k=3, \ell=25: \quad\left(\mathcal{E}_{3,28} \otimes \mathcal{A}_{25}\right) M(101)$ (interchange second and third components);

(sp.11) $k=8, \ell=28: \quad \mathcal{E}_{8,28} \otimes \mathcal{D}_{36}$

(sp.12) $k=8, \ell=20: \quad \mathcal{E}_{8,28} \otimes \mathcal{D}_{20}$ (interchange second and third components).

When $\ell=1$, we get the invariants listed in Sect.3.1 with no redundancies. However the identity $\mathcal{D}_{2}=\mathcal{A}_{2}$ means we do get some repetition when $\ell=2$ (the $\ell=2$ cosets include all of the $N=1$ superconformal minimal models). We conjecture that this list gives all physical invariants of $s u(2)_{k} \oplus s u(2)_{\ell} \oplus s u(2)_{k+\ell}$ satisfying (2.8); in the next subsection we prove this for certain $k, \ell$ (we have also done this for all $k$, when $\ell=2$ ).

\subsection{The small gcd case}

The only class of $s u(2)$ cosets which is classified is that with $\ell=1$ [4]. In this subsection we will exploit other results from [7] which will permit us to obtain many more classifications. In particular, there we found (among other things) all invariants of $s u(2)_{k_{1}} \oplus \cdots \oplus s u(2)_{k_{r}}$ when for each $i \neq j, g c d\left(k_{i}+2, k_{j}+2\right) \leq 3$. So from this we immediately get the list of all $s u(2)_{k} \oplus s u(2)_{\ell} / s u(2)_{k+\ell}$ physical invariants when the greatest common divisors $\operatorname{gcd}(k+2, \ell+2), \operatorname{gcd}(k+2, \ell)$, and $\operatorname{gcd}(k, \ell+2)$ are all $\leq 3$. (The relevant property of the numbers $a \leq 3$ is that the only numbers coprime to $2 a$ are $\equiv \pm 1$ $(\bmod 2 a)$.) Note that for a given $k$, there are infinitely many $\ell$ satisfying those three $g c d$ conditions - in fact a positive density of such $\ell$.

This argument in [7] makes use of some additional properties WZNW partition functions must satisfy [20]. We can avoid using these here, because we have only three copies of $s u(2)$, and because (2.8) holds. As usual, we will restrict attention here to the cosets without fixed points, i.e. where at least one of $k$ and $\ell$ is odd. With a little more work, this restriction can be lifted.

The proof of the following theorem makes use of certain useful lemmas and techniques (e.g. the parity rule) developed in several earlier papers. We state these explicitly in Sects.4 and 5 , in the context of $s u(3)$, in the course of proving the more difficult Thm.2, but to avoid unnecessary duplication we will not give their $s u(2)$ translations here. For that, see in particular [7].

Theorem 1. Choose any $k, \ell$, not both even, for which $\operatorname{gcd}(k+2, \ell+2), \operatorname{gcd}(k+2, \ell), \operatorname{gcd}(k$, $\ell+2)$ are all $\leq 3$. Then the complete list of all physical invariants of $s u(2)_{k} \oplus s u(2)_{\ell} \oplus$ su(2) $k+\ell$ satisfying (2.8) is (sc.1), (sc.2), (e.1) and (e.3).

Proof The argument will follow as closely as possible the proof of Thm.7 in [7]. Since the detailed argument is so similar to others (e.g. analyzing the special cases $k_{1}=10,16,28$ reduces to arguments found in Sect.5.3 below, and Sect.6 of [10]), we will be somewhat sketchy here.

Exactly one of $k, \ell, k+\ell$ will be even; let $k_{1}$ denote this even level, and let $k_{2} \leq k_{3}$ denote the other two, and write $k_{i}^{\prime}=k_{i}+2$. Let $M$ denote any physical invariant of $s u(2)_{k_{1}} \oplus s u(2)_{k_{2}} \oplus s u(2)_{k_{3}}$. Write $a=\left(a_{1}, a_{2}, a_{3}\right)$ etc for its weights, $\rho$ for the weight 
$(1,1,1)$, and $S_{a b}$ for the $S$-matrix. In particular we first find in Sect.6 of [7] the consequences of the so-called parity rule (in fact the reason for our $g c d$ conditions is precisely to maximize the effectiveness of this parity rule). We get the following selection rules:

$$
\begin{aligned}
M_{a b} \neq 0 & \Rightarrow b_{2} \in\left\{a_{2}, k_{2}^{\prime}-a_{2}\right\}, \quad b_{3} \in\left\{a_{3}, k_{3}^{\prime}-a_{3}\right\} ; \\
M_{a b} \neq 0 & \Rightarrow a_{1}^{2} \equiv b_{1}^{2}\left(\bmod k_{1}^{\prime}\right) \\
M_{a b} \neq 0, a_{1}=1, k_{1} \neq 10,28 & \Rightarrow b_{1} \in\left\{1, k_{1}^{\prime}-1\right\} \\
M_{a b} \neq 0, a_{1}=1, k_{1}=10 & \Rightarrow b_{1} \in\{1,5,7,11\} \\
M_{a b} \neq 0, a_{1}=1, k_{1}=28 & \Rightarrow b_{1} \in\{1,11,19,29\}
\end{aligned}
$$

Eq. (3.5a) is Lemma 3(a) in [7], (3.5b) is T-invariance using (3.5a), and (3.5c)-(3.5e) are Lemma 3(b) in [7], using (3.5b).

Let $\mathcal{R}_{L}=\left\{a \mid M_{a \rho} \neq 0\right\}, \mathcal{R}_{R}=\left\{b \mid M_{\rho b} \neq 0\right\}$. Write $\mathcal{J}_{L}$ for the set of all simple currents $J$ such that $J \rho \in \mathcal{R}_{L}$; define $\mathcal{J}_{R}$ similarly. Much is known about the sets $\mathcal{R}_{L, R}$ and $\mathcal{J}_{L, R}$ - see e.g. [7], [10], and Lemmas 4 and 5 in Sect.5 of this paper. For instance the cardinalities $\left\|\mathcal{J}_{L}\right\|$ and $\left\|\mathcal{J}_{R}\right\|$ must be equal, and $\mathcal{J}_{L, R}$ both are groups (under componentwise addition mod 2). Also each norm $J^{2}\left(\right.$ see $(3.1 b)$ ) in $\mathcal{J}_{L, R}$ must be 0 , and $M_{J a, J^{\prime} b}=M_{a b}$ $\forall J \in \mathcal{J}_{L}, J^{\prime} \in \mathcal{J}_{R}$. Also, $S_{a, J b}=-1^{J \cdot(a-\rho)} S_{a b}$, and for $J \in \mathcal{J}_{L}, J \cdot(a-\rho) \equiv 0$ if $M_{a b} \neq 0$ for some $b$. Recall the definition of simple current invariant, given in (3.2); all simple current invariants for $s u(2)_{\ell_{1}} \oplus \cdots \oplus s u(2)_{\ell_{r}}$ are explicitly known, and in our case will lie in (sc.1) or (sc.2).

Case 1: Consider first the case where all $a \in \mathcal{R}_{L} \cup \mathcal{R}_{R}$ satisfy $a_{1} \in\left\{1, k_{1}^{\prime}-1\right\}-$ i.e. $\mathcal{J}_{L} \rho=$ $\mathcal{R}_{L}$ and $\mathcal{J}_{R} \rho=\mathcal{R}_{R}$. By (3.5c), this is the case most of the time. a is called a fixed point of $\mathcal{J}_{L}$ if $J a=a$ for some $J \in \mathcal{J}_{L}, J \neq 0$. Note that $\mathcal{J}_{L}$ has a fixed point iff $(1,0,0) \in \mathcal{J}_{L}$, in which case $a$ is a fixed point iff $a_{1}=k_{1}^{\prime} / 2$. Similar comments apply to $\mathcal{J}_{R}$.

Suppose $M_{a b} \neq 0$, and $a$ is not a fixed point of $\mathcal{J}_{L}$, and $b$ is not one of $\mathcal{J}_{R}$. Without loss of generality suppose $S_{a \rho} \leq S_{b \rho}$. Then from the various results about $\mathcal{J}_{L, R}$ and $S$ given earlier, we get

$$
\left\|\mathcal{J}_{R}\right\| M_{a b} S_{b \rho} \leq(M S)_{a \rho}=(S M)_{a \rho}=\left\|\mathcal{J}_{L}\right\| S_{a \rho} .
$$

Since $\left\|\mathcal{J}_{L}\right\|=\left\|\mathcal{J}_{R}\right\|$, this tells us that $M_{a d}=1$ iff $d \in \mathcal{J}_{R} b$, otherwise it equals 0. It also tells us that $S_{b \rho}=S_{a \rho}$, i.e. (using (3.5a)) $b_{1} \in\left\{a_{1}, k_{1}^{\prime}-a_{1}\right\}$. Similarly, we get that $M_{c b}=1$ iff $c \in \mathcal{J}_{L} a$, otherwise it equals 0 . Thus if there are no fixed points, $M$ must satisfy (3.2) and we are done. We may assume then that $k_{1} \equiv 0(\bmod 4)$ and $(1,0,0) \in \mathcal{J}_{R}$, say. We may also assume $k_{1}>4$, since $(3.5 a),(3.5 b)$ force $(3.2)$ if $k_{1}=4$.

Now put $x=(3,1,1)$ and choose $y$ so that $M_{x y} \neq 0 . x$ is not a fixed point of $\mathcal{J}_{L}$; for now assume $y$ is not one of $\mathcal{J}_{R}$. Then by the previous paragraph, $y_{1} \in\left\{3, k_{1}^{\prime}-3\right\}$. Using this, we can prove (3.2) holds for all $a, b$, as follows. Suppose $M_{a b} \neq 0$, where $a_{1} \neq k_{1}^{\prime} / 2$, but $b$ is a fixed point of $\mathcal{J}_{R}$. Then $S M=M S$ applied to $(a, \rho)$ and $(a, y)$ gives us

$$
1=2 \sin \left(\pi a_{1} / k_{1}^{\prime}\right), \quad \pm 1=2 \sin \left(3 \pi a_{1} / k_{1}^{\prime}\right),
$$

which are incompatible. This implies $M$ is a simple current invariant, and we are done. 
If instead $y$ defined in the previous paragraph is a fixed point of $\mathcal{J}_{R}$, then $y_{1}=k_{1}^{\prime} / 2$, and $(3.5 b)$ forces $k_{1}=16$. Suppose $M_{a b} \neq 0$. Then (3.5b) tells us that if $a_{1} \notin\{3,9,15\}$, then $b_{1} \in\left\{a_{1}, k_{1}^{\prime}-a_{1}\right\}$, and if $a_{1} \in\{3,9,15\}$ then $b_{1} \in\{3,9,15\}$ (incidently, by Lemma 4 (b) this forces $\left.(1,0,0) \in \mathcal{J}_{L}\right)$. Suppose $a_{1}=3$ but $b_{1} \neq 9$; then from $(S M)_{x b}=(M S)_{x b}$ we get $2 S_{a x}= \pm S_{a y}$, i.e. $2 \sin (9 \pi / 18)= \pm \sin (27 \pi / 18)$, which is impossible.

This proves (using Lemmas $4(\mathrm{~b})$ and $5(\mathrm{a})$ ) that $a_{1}$ and $b_{1}$ are related to each other independently of the values of $a_{2}, a_{3}, b_{2}, b_{3}$. The reverse can also be seen to hold, using T-invariance, $(3.5 a)$, and (2.8). Thus $M$ will be the tensor product of $\mathcal{E}_{16}$ with some simple current invariant of $s u(2)_{k_{2}} \oplus s u(2)_{k_{3}}$, and will be listed in (e.3).

Case 2: By $(3.5 c)$, Case 1 handled all values of $k_{1}$ except 10 and 28. These exceptional levels use familiar arguments (see e.g. Sect.5.3) and we will not repeat them here. The idea is to first find the possibilities for the values of $M_{\rho b}$ and $M_{a \rho}$ (this is done in the proof of Thm.7 in [7]), and then use Lemmas 4(b) and 5 and modular invariance (particularly the relation $S M=M S$ ) to find the other values of $M . \quad$ QED

\section{The physical invariants of $s u(3)_{k} \oplus s u(3)_{1} / s u(3)_{k+1}$}

The last section was an illustration of carrying over known WZNW classifications directly to the GKO classification. Unfortunately, there are few examples of WZNW classifications, even for simple $g$. One of these is $g=s u(3)$. Our goal in this section and the next is to prove the following theorem, which we learned in Sect.2.3 solves the classification problem for the cosets $s u(3)_{k} \oplus s u(3)_{1} / s u(3)_{k+1}, \forall k$ :

Theorem 2. The list of all physical invariants of $s u(3)_{k} \oplus s u(3)_{k+1}$ satisfying (2.11c) is given in the Table.

In the Table and throughout the remainder of this paper, $\overline{\mathcal{A}}_{k}, \overline{\mathcal{D}}_{k}$ and $\overline{\mathcal{E}}_{k}$ denote the physical invariants of $s u(3)_{k}$, and are explicitly given in [8]. The simple current invariants $\bar{M}\left(A_{k} A_{k+1}\right)$ are defined in (4.4), and their conjugation ${ }^{c}\left[\bar{M}\left(A_{k} A_{k+1}\right)\right]^{c}$ is defined in $(4.10 a)$. The invariants of $s u(3)_{k} \oplus s u(3)_{1} / s u(3)_{k+1}$ were discussed in [1,5], but their lists are very incomplete.

Sections 4 and 5 are devoted to proving Thm.2. Our task in this section is to find all physical invariants which are automorphisms of the unextended chiral algebra, but we will begin with some general observations which will also be useful in the following section.

\subsection{General comments}

As usual write $\lambda=\left(\lambda_{1}, \lambda_{2}\right)$ for an $s u(3)$ weight, $k^{\prime}=k+3$ for the height, $t(\lambda) \equiv$ $\lambda_{1}-\lambda_{2}(\bmod 3), A_{k}$ for the simple current taking $\lambda$ to $A_{k} \lambda=\left(k^{\prime}-\lambda_{1}-\lambda_{2}, \lambda_{1}\right)$ and $C$ for the conjugation $C \lambda=\left(\lambda_{2}, \lambda_{1}\right)$. Write $\mathcal{P}(k)$ for $P_{++}(s u(3), k) \times P_{++}(s u(3), k+1)$. Together, $A_{k}$ (which is order 3 ) and $C$ (which is order 2 ) generate the order 6 group of outer automorphisms $\mathcal{O}^{k}$ of affine $s u(3)$. Let $\mathcal{O}^{k} \lambda$ denote the orbit $\left\{C^{a} A_{k}^{b} \lambda \mid a=0,1 ; b=0,1,2\right\}$ of $\lambda$ by this group. Also, let $\mathcal{O}_{0}^{k} \lambda$ be the orbit $\left\{A_{k}^{b} \lambda \mid b=0,1,2\right\}$ of $\lambda$ by the simple currents. For example, $\mathcal{O}^{k} \rho=\mathcal{O}_{0}^{k} \rho=\{(1,1),(k+1,1),(1, k+1)\}$. 
What we need to consider is $s u(3)_{k} \oplus s u(3)_{k+1}$. Note first that the heights $k^{\prime}$ and $k^{\prime}+1$ are coprime, so the two summands are almost independent. For example, $T$-invariance implies that if $M_{\lambda \mu, \kappa \nu} \neq 0$ for any invariant $M$, then

$$
3 \lambda^{2} \equiv 3 \kappa^{2}\left(\bmod 2 k^{\prime}\right) ; \quad 3 \mu^{2} \equiv 3 \nu^{2}\left(\bmod 2 k^{\prime}+2\right) .
$$

Also very important is the parity rule of $[6]$ (=the arithmetical symmetry of $[22])$. For $s u(3)_{k} \oplus s u(3)_{k+1}$ it reduces to the following. Let $\lambda \in P(s u(3))$. There exists a unique weight, call it $[\lambda]_{k}$, lying in both $P_{+}(s u(3), k+3)$ and the orbit of $\lambda$ by the affine Weyl group. If $[\lambda]_{k}$ also lies in $P_{++}(s u(3), k)$, then there exists a unique (finite) Weyl automorphism $\omega$ and vector $\alpha \in P(s u(3))^{*}$, the root lattice of $s u(3)$, such that $[\lambda]_{k}=\omega\left(\lambda+k^{\prime} \alpha\right)$; in this case we can define the parity $p_{k}(\lambda)$ of $\lambda$ to be the parity $\epsilon(\omega)=\operatorname{det}(\omega)$. The parity rule tells us that for any invariant $M$, any integer $\ell$ coprime to $3 k^{\prime}\left(k^{\prime}+1\right)$, and any $(\lambda \mu),(\kappa \nu) \in \mathcal{P}(k)$, we have

$$
M_{\lambda \mu, \kappa \nu}=p_{k}(\ell \lambda) p_{k+1}(\ell \mu) p_{k}(\ell \kappa) p_{k+1}(\ell \nu) M_{\lambda^{\prime} \mu^{\prime}, \kappa^{\prime} \nu^{\prime}}
$$

where $\lambda^{\prime}=[\ell \lambda]_{k}, \mu^{\prime}=[\ell \mu]_{k+1}, \kappa^{\prime}=[\ell \kappa]_{k}$ and $\nu^{\prime}=[\ell \nu]_{k+1}$.

Because $k^{\prime}$ and $k^{\prime}+1$ are coprime, this simplifies a little. Given any $\ell_{1}$ and $\ell_{2}$, there will be an $\ell$ satisfying

$$
\ell \equiv \ell_{1}\left(\bmod 3 k^{\prime}\right) \text { and } \ell \equiv \ell_{2}\left(\bmod 3\left(k^{\prime}+1\right)\right)
$$

iff $\ell_{1} \equiv \ell_{2}(\bmod 3)$. Let $\ell_{1}$ be any integer coprime to $3 k^{\prime}$, and choose $\ell_{2}= \pm 1$ so that $\ell_{1} \equiv \ell_{2}$ $(\bmod 3)$. Find any $\ell$ satisfying $(4.2 b)$. It is easy to see that, for any $\mu, \nu \in P_{++}(s u(3), k+1)$, $p_{k+1}(\ell \mu)=\ell_{2}=p_{k+1}(\ell \nu)$. Then (4.2a) implies the following equation, familiar from [8]. Let $M$ be any physical invariant, and suppose $M_{\lambda \mu, \kappa \nu} \neq 0$. By $\{x\}$ we mean the unique number $y$ satisfying both $0 \leq y<k^{\prime}$ and $y \equiv x\left(\bmod k^{\prime}\right)$. Then for all integers $\ell_{1}$ coprime to $3 k^{\prime}$,

$$
\left\{\ell_{1} \lambda_{1}\right\}+\left\{\ell_{1} \lambda_{2}\right\}<k^{\prime} \text { iff }\left\{\ell_{1} \kappa_{1}\right\}+\left\{\ell_{1} \kappa_{2}\right\}<k^{\prime}
$$

a similar statement holds for $\mu$ and $\nu$, with $k^{\prime}$ replaced with $k^{\prime}+1$. We will later need other consequences of the parity rule $(4.2 a)$.

Two consequences of (4.1) and (4.3) have already been drawn in the literature. In [16] in a completely different context (this paper was "discovered" and brought into the context of modular invariants by [22]), it was proved that:

Lemma 1. When $k^{\prime}$ is coprime to $6, \lambda$ and $\kappa$ satisfy (4.3) iff $\kappa \in \mathcal{O}^{k} \lambda$.

Of course an analogous statement holds for $\mu, \nu$ and $k^{\prime}+1$. Eq.(4.1) was not used in this derivation. This remarkable result has the flaw that it only holds for some $k$. When $k^{\prime}$ and 6 are not coprime, the situation becomes considerably more complicated, and no classification of the solutions $\lambda, \kappa$ to (4.3) is known for general $k$ (it would likely be very messy and probably useless). However, if we also use (4.1) and restrict ourselves to the special case $\kappa=\rho$, all $\lambda$ solving (4.3) are known, for all $k$ (this is Prop.1 in [10]):

Lemma 2. The set of all solutions $\lambda$ to (4.1) and (4.3), for $\kappa=\rho$, is:

(a) for $k \equiv 0,2,3(\bmod 4), k \neq 15: \lambda \in \mathcal{O}_{0}^{k} \rho$; 
(b) for $k \equiv 1(\bmod 4), k \neq 9,21,57: \lambda \in \mathcal{O}_{0}^{k} \rho \cup \mathcal{O}_{0}^{k}((k+1) / 2,(k+1) / 2)$;

(c) for $k=9,15,21,57$, respectively, $\lambda$ lies in

$$
\begin{aligned}
& \mathcal{O}_{0}^{9} \rho \cup \mathcal{O}^{9}(3,3)_{0} \cup \mathcal{O}_{0}^{9}(5,5) \\
& \mathcal{O}_{0}^{15} \rho \cup \mathcal{O}^{15}(1,4) \\
& \mathcal{O}_{0}^{21} \rho \cup \mathcal{O}_{0}^{21}(5,5) \cup \mathcal{O}_{0}^{21}(7,7) \cup \mathcal{O}_{0}^{21}(11,11) \\
& \mathcal{O}_{0}^{57} \rho \cup \mathcal{O}_{0}^{57}(11,11) \cup \mathcal{O}_{0}^{57}(19,19) \cup \mathcal{O}_{0}^{57}(29,29)
\end{aligned}
$$

Some useful results concerning the modular $S$-matrix $S^{(k)}$ of $s u(3)_{k}$ are Claims 1,2 , 8 in $[10]$ :

Lemma 3. (a) Suppose $S_{\lambda \rho}^{(k)}=S_{\kappa \rho}^{(k)}$ and $\left\{\lambda_{1}, \lambda_{2}, k^{\prime}-\lambda_{1}-\lambda_{2}\right\} \cap\left\{\kappa_{1}, \kappa_{2}, k^{\prime}-\kappa_{1}-\kappa_{2}\right\} \neq\{\}$. Then $\kappa \in \mathcal{O}^{k} \lambda$.

(b) For any $\lambda, a, b, S_{(1,2), C^{a} A^{b} \lambda}^{(k)}=S_{(1,2), \lambda}^{(k)}$ iff $C^{a} A^{b} \lambda=\lambda$.

(c) For any $\lambda, a, b, S_{(1,4), C^{a} A^{b} \lambda}^{(k)}=S_{(1,4), \lambda}^{(k)}$ iff $C^{a} \lambda \in\left\{\lambda, A \lambda, A^{2} \lambda\right\}$.

Finally, we will define [12] the physical invariant $\bar{M}(J)$ of $s u(3)_{k} \oplus s u(3)_{k+1}$ associated with the simple current $J=A_{k}^{a} A_{k+1}^{b}$, for $a, b$ not both divisible by 3 (compare $(3.3 a)$ ):

$$
[\bar{M}(J)]_{\lambda \mu, \kappa \nu}=\sum_{m=0}^{2} \delta_{J^{m}(\lambda \mu), \kappa \nu} \delta_{3}\left[a t(\lambda)+b t(\mu)-m\left(k a^{2}+(k+1) b^{2}\right)\right] .
$$

\subsection{The automorphism invariants of $s u(3)_{k} \oplus s u(3)_{k+1}$}

A natural first step is to find all automorphism(=permutation) invariants corresponding to that direct sum. The hardest part of this (Claim 3 below) will be to show that "locally" the automorphism invariant acts like an outer automorphism.

By an automorphism invariant we mean a physical invariant $M=M^{\sigma}$ with

$$
\left(M^{\sigma}\right)_{\lambda \mu, \kappa \nu}=\delta_{\kappa \nu, \sigma(\lambda \mu)}
$$

where $\sigma$ is a permutation of $\mathcal{P}(k)$. The entries of $M^{\sigma}$ will be a bunch of 0 's and 1's, with exactly one ' 1 ' on each row and column. Of course, $M_{\rho \rho, \rho \rho}^{\sigma}=1$ means that $\sigma(\rho \rho)=(\rho \rho)$.

Commutation of $M^{\sigma}$ with $S^{(k, k+1)}=S^{(k)} \otimes S^{(k+1)}$ means that

$$
\begin{gathered}
S_{\lambda \kappa}^{(k)} S_{\mu \nu}^{(k+1)}=S_{\lambda^{\prime} \kappa^{\prime}}^{(k)} S_{\mu^{\prime} \nu^{\prime}}^{(k+1)}, \\
N_{\lambda \kappa \alpha}^{(k)} N_{\mu \nu \beta}^{(k+1)}=N_{\lambda^{\prime} \kappa^{\prime} \alpha^{\prime}}^{(k)} N_{\mu^{\prime} \nu^{\prime} \beta^{\prime}}^{(k+1)},
\end{gathered}
$$

where $(\lambda \mu),(\kappa \nu),(\alpha \beta) \in \mathcal{P}(k)$, where $\sigma(\lambda \mu)=\left(\lambda^{\prime} \mu^{\prime}\right), \sigma(\kappa \nu)=\left(\kappa^{\prime} \nu^{\prime}\right)$ and $\sigma(\alpha \beta)=\left(\alpha^{\prime} \beta^{\prime}\right)$, and where $N^{(k)}$ are the fusion coefficients for $s u(3)_{k}$ (similarly for $\left.s u(3)_{k+1}\right)$. By Verlinde's formula, we know (4.5a) implies (4.5b). 
The entries of $S^{(k)}$ are given by the Kac-Peterson formula [14], while those of $N^{(k)}$ (for $s u(3))$ were computed in [2]. Two important special cases are:

$$
\begin{aligned}
S_{\rho \lambda}^{(k)} & =\frac{2}{\sqrt{3} k^{\prime}}\left\{s\left(2 \lambda_{1}\right)+s\left(2 \lambda_{2}\right)+s\left(2 \lambda_{0}\right)\right\}=\frac{8}{\sqrt{3} k^{\prime}} s\left(\lambda_{1}\right) s\left(\lambda_{2}\right) s\left(\lambda_{0}\right)>0, \\
N_{\lambda \lambda \lambda}^{(k)} & =\min _{i=0,1,2}\left\{\lambda_{i}\right\}
\end{aligned}
$$

where $\lambda_{0}=k^{\prime}-\lambda_{1}-\lambda_{2}$ and $s(x)=\sin \left(\pi x / k^{\prime}\right)$. From $(4.6 a)$ we see that $\sqrt{3} S_{\rho \lambda}^{(k)}$ is a polynomial over $\mathbf{Q}$ in $\xi_{k^{\prime}}=\exp \left(2 \pi i / k^{\prime}\right)$. But we know (see e.g. [25]) that the roots of unity $\xi_{m}$ and $\xi_{n}$ are algebraically independent over $\mathbf{Q}$, whenever $m$ and $n$ are coprime. Using (4.5a) and the fact that $k^{\prime}$ and $k^{\prime}+1$ are coprime, what this means is that

$$
\sigma(\lambda, \mu)=\left(\lambda^{\prime}, \mu^{\prime}\right) \Rightarrow \frac{S_{\rho \lambda}^{(k)}}{S_{\rho \lambda^{\prime}}^{(k)}}=\frac{S_{\rho \mu^{\prime}}^{(k+1)}}{S_{\rho \mu}^{(k+1)}} \in \mathbf{Q} .
$$

Another convenient formula for the S-matrix of $s u(3)_{k}$ is, for any weights $\lambda, \kappa$,

$$
S_{\lambda, C \kappa}^{(k)}=\left(S_{\lambda \kappa}^{(k)}\right)^{*}
$$

Our first task will be to prove:

Claim 3. Any automorphism invariant $M^{\sigma}$ for $s u(3)_{k} \oplus s u(3)_{k+1}$ acts locally like an outer automorphism. In other words, choose any $(\lambda \mu) \in \mathcal{P}(k)$ and write $\sigma(\lambda \mu)=\left(\lambda^{\prime} \mu^{\prime}\right)$; then both $\lambda^{\prime} \in \mathcal{O}^{k} \lambda$ and $\mu^{\prime} \in \mathcal{O}^{k+1} \mu$.

Proof Suppose $(\lambda \mu)$ has $\lambda^{\prime} \in \mathcal{O}^{k} \lambda$. Then eqs.(4.5) and (4.6) tell us that

$$
S_{\rho \mu}^{(k+1)}=S_{\rho \mu^{\prime}}^{(k+1)}, \quad N_{\mu \mu \mu}^{(k+1)}=N_{\mu^{\prime} \mu^{\prime} \mu^{\prime}}^{(k+1)}
$$

Lemma 3(a) then tells us that $\mu^{\prime} \in \mathcal{O}^{k+1} \mu$. Thus $\lambda^{\prime} \in \mathcal{O}^{k} \lambda$ iff $\mu^{\prime} \in \mathcal{O}^{k+1} \mu$.

But for $k \equiv 2,4(\bmod 6)$ Lemma 1 tells us that $\lambda^{\prime} \in \mathcal{O}^{k} \lambda$, and for $k \equiv 1,3(\bmod 6)$ it tells us that $\mu^{\prime} \in \mathcal{O}^{k+1} \mu$. This concludes the proof of our Claim for $k \equiv 1,2,3,4(\bmod 6)$. It remains to consider $k \equiv 0,5,6,11(\bmod 12)$. For this purpose, we will make use of the following important fact:

For any $(\lambda \mu),(\kappa \nu) \in \mathcal{P}(k)$ and integers $a, b$,

$$
\sigma(\rho \mu)=\left(A^{a} \rho, \nu\right) \text { and } \sigma(\lambda \rho)=\left(\kappa, A^{b} \rho\right) \Rightarrow \sigma(\lambda \mu)=\left(A^{a} \kappa, A^{b} \nu\right) .
$$

Eq.(4.8a) holds for any level $k$, and analogues will hold for all algebras $g$. To see it, write $\sigma(\lambda \mu)=\left(\lambda^{\prime} \mu^{\prime}\right)$; then $\lambda^{\prime}, \mu^{\prime}$ are the unique weights satisfying

$$
S_{\alpha \lambda}^{(k)} S_{\beta \mu}^{(k+1)}=S_{\alpha^{\prime} \lambda^{\prime}}^{(k)} S_{\beta^{\prime} \mu^{\prime}}^{(k+1)}
$$

for all $(\alpha \beta) \in \mathcal{P}(k)$, where $\sigma(\alpha \beta)=\left(\alpha^{\prime} \beta^{\prime}\right)$. (This follows e.g. from orthogonality of the rows of $S^{(k, k+1)}$.) Using $(2.2 c)$, the two hypotheses in $(4.8 a)$ tell us

$$
S_{\alpha \rho}^{(k)} S_{\beta \mu}^{(k+1)}=\omega^{a t\left(\alpha^{\prime}\right)} S_{\alpha^{\prime} \rho}^{(k)} S_{\beta^{\prime} \mu^{\prime}}^{(k+1)}, \quad S_{\alpha \lambda}^{(k)} S_{\beta \rho}^{(k+1)}=\omega^{b t\left(\beta^{\prime}\right)} S_{\alpha^{\prime} \lambda^{\prime}}^{(k)} S_{\beta^{\prime} \rho}^{(k+1)},
$$


where $\omega=\exp (2 \pi i / 3)$. Multiplying these and dividing by the corresponding equation for $\sigma(\rho \rho)=(\rho \rho)$ gives us $(4.8 b)$ with $\left(\lambda^{\prime} \mu^{\prime}\right)=\left(A^{a} \kappa, A^{b} \nu\right)$, completing the proof of $(4.8 a)$.

One immediate consequence of $(4.8 a)$ is that if we know that Claim 3 holds for $(\lambda \rho)$, and $(\rho \mu)$, then it holds for $(\lambda \mu)$.

Look first at $k \equiv 11(\bmod 12)$. Choose any $(\lambda \mu) \in \mathcal{P}(k)$, and write $\sigma(\lambda \rho)=\left(\lambda^{\prime} \rho^{\prime}\right)$ and $\sigma(\rho \mu)=\left(\rho^{\prime \prime} \mu^{\prime}\right)$. By Lemma 2(a) we read that $\rho^{\prime} \in \mathcal{O}_{0}^{k} \rho$ and $\rho^{\prime \prime} \in \mathcal{O}_{0}^{k+1} \rho$. By the conclusion of the paragraph containing (4.7), this forces both $\lambda^{\prime} \in \mathcal{O}^{k} \lambda$ and $\mu^{\prime} \in \mathcal{O}^{k+1} \mu$. By the previous paragraph, this concludes the proof of Claim 3 for these $k$.

The identical argument and conclusion applies to $k \equiv 6(\bmod 12)$. For $k \equiv 0,5(\bmod$ 12), respectively, we have to consider the additional possibilities that $\rho^{\prime} \in \mathcal{O}_{0}^{k+1}((k+$ $2) / 2,(k+2) / 2)$ and $\rho^{\prime \prime} \in \mathcal{O}_{0}^{k}((k+1) / 2,(k+1) / 2)$. Consider without loss of generality the latter case. Then $(4.6 c)$ becomes the statement that

$$
\frac{S_{\rho \rho}^{(k)}}{S_{\rho \rho^{\prime \prime}}^{(k)}}=\frac{\sin \left(\pi / k^{\prime}\right) \sin \left(\pi / k^{\prime}\right) \sin \left(\pi(k+1) / k^{\prime}\right)}{\sin \left(\pi(k+1) / 2 k^{\prime}\right) \sin \left(\pi(k+1) / 2 k^{\prime}\right) \sin \left(2 \pi / k^{\prime}\right)}=\frac{2}{1+\cos \left(2 \pi / k^{\prime}\right)}-1
$$

is a rational number. In other words, $k^{\prime}$ must satisfy

$$
\cos \left(2 \pi / k^{\prime}\right) \in \mathbf{Q}
$$

For $n>2$, the degree $[\mathbf{Q}(\cos (2 \pi / n)): \mathbf{Q}]$ of the extension of $\mathbf{Q}$ by $\cos (2 \pi / n)$ equals $[\mathbf{Q}(\exp (2 \pi i / n)): \mathbf{Q}] /[\mathbf{Q}(\exp (2 \pi i / n)): \mathbf{Q}(\cos (2 \pi / n))]=\phi(n) / 2$, where $\phi(n)$ is the number of $1 \leq \ell<n$ coprime to $n$. Therefore $(4.9 b)$ holds iff $\phi\left(k^{\prime}\right) \leq 2$, i.e. iff $k^{\prime}=1,2,3,4,6 .^{3}$

But none of these solutions to $(4.9 b)$ satisfy $k^{\prime}=k+3 \equiv 8(\bmod 12)$. This contradiction means that $\rho^{\prime \prime}$ must lie in $\mathcal{O}^{k} \rho$. The familiar argument now forces $\mu^{\prime} \in \mathcal{O}^{k+1} \mu$, completing the proof of Claim 3 for $k \equiv 5(\bmod 12)$. The argument for $k \equiv 0(\bmod 12)$ is identical. QED

Given any physical invariant $M$, define the conjugations $M^{c},{ }^{c} M,{ }^{c} M^{c}$ by

$$
\left(M^{c}\right)_{\lambda \mu, \kappa \nu}=M_{\lambda \mu, \kappa C \nu}, \quad\left({ }^{c} M\right)_{\lambda \mu, \kappa \nu}=M_{\lambda \mu, C \kappa \nu}, \quad\left({ }^{c} M^{c}\right)_{\lambda \mu, \kappa \nu}=M_{\lambda \mu, C \kappa C \nu}
$$

Then each of these conjugations will also be a physical invariant.

Claim 4. $\quad M$ is an automorphism invariant of $s u(3)_{k} \oplus s u(3)_{k+1}$ iff one of the 4 conjugations (4.10a) of $M$, call it $M^{\sigma^{\prime}}$, satisfies:

$$
\forall(\lambda \mu) \in \mathcal{P}(k), \quad \sigma^{\prime}(\lambda \mu)=\left(A^{a t(\lambda)+b t(\mu)} \lambda, A^{c t(\lambda)+d t(\mu)} \mu\right),
$$

for fixed $(a, b, c, d)$, independent of $\lambda, \mu$. Moreover,

(a) for $k \equiv 0(\bmod 3),(a, b, c, d) \in\{(0,0,0,0),(1, \pm 1, \mp 1,0),(0,0,0,1),(1, \pm 1, \pm 1,1)\}$,

(b) for $k \equiv 1(\bmod 3),(a, b, c, d) \in\{(0,0,0,0),(0,0,0,-1),(1,0,0,0),(1,0,0,-1)\}$,

(c) for $k \equiv 2(\bmod 3),(a, b, c, d) \in\{(0,0,0,0),(-1, \pm 1, \pm 1,-1),(-1,0,0,0),(0, \pm 1, \mp 1,-1)\}$.

3 We thank Antoine Coste for this proof that (4.9b) implies $\mathrm{k}^{\prime}=1,2,3,4,6$. 
Proof First, it is necessary to verify that all the quadruples $(a, b, c, d)$ listed in Claim 4 give rise to automorphism invariants. It suffices to verify $T$-invariance and that $S_{\lambda \mu, \kappa \nu}^{(k, k+1)}=$ $S_{\sigma^{\prime}(\lambda \mu), \sigma^{\prime}(\kappa \nu)}^{(k, k+1)}$. This is straightforward using eqs. $(2.2 b),(2.2 c)$.

Let $M=M^{\sigma}$. Since $C \rho=\rho$, Claim 3 tells us $\sigma(\rho,(1,2))=\left(A^{w} \rho, C^{u} A^{x}(1,2)\right)$ and $\sigma((1,2), \rho)=\left(C^{v} A^{y}(1,2), A^{z} \rho\right)$. Consider the conjugation $M^{\prime}$ of $M$ with $u=v=0$. Eqs. $(4.5 a)$ and $(2.2 c)$ give us the following conditions of $w, x, y, z$ :

$$
\begin{aligned}
& S_{\rho \rho}^{(k)} S_{(1,2),(1,2)}^{(k+1)}=S_{A^{w} \rho, A^{w} \rho^{\prime}}^{(k)} S_{A^{x}(1,2), A^{x}(1,2)}^{(k+1)}: \quad w^{2} k+x+x^{2}(k+1) \equiv 0(\bmod 3), \\
& S_{(1,2),(1,2)}^{(k)} S_{\rho \rho}^{(k+1)}=S_{A^{y}(1,2), A^{y}(1,2)}^{(k)} S_{A^{z} \rho, A^{z} \rho}^{(k+1)}: \quad y^{2} k+y+z^{2}(k+1) \equiv 0(\bmod 3), \\
& S_{\rho,(1,2)}^{(k)} S_{(1,2), \rho}^{(k+1)}=S_{A^{w} \rho, A^{y}(1,2)}^{(k)} S_{A^{x}(1,2), A^{z} \rho}^{(k+1)}: \quad w y k-w+x z(k+1)-z \equiv 0(\bmod 3) .
\end{aligned}
$$

Here we are exploiting the facts that $S_{\rho \lambda}^{(\ell)} \neq 0$ (see $(4.6 a)$ ) and $S_{(1,2),(1,2)}^{(\ell)} \neq 0$ (see Lemma $3(\mathrm{~b}))$. It is easy to find all solutions $(w, x, y, z)$ to (4.11), and to verify that each one corresponds to some $M^{\sigma^{\prime}}$ listed in the Claim.

In other words, the automorphism invariant $M^{\sigma^{\prime \prime}}=M^{\prime}\left(M^{\sigma^{\prime}}\right)^{-1}$ has $\sigma^{\prime \prime}(\rho,(1,2))=$ $(\rho,(1,2))$ and $\sigma^{\prime \prime}((1,2), \rho)=((1,2), \rho)$. By Claim 3 we may write $\sigma^{\prime \prime}(\lambda, \mu)=\left(C^{e} A^{f} \lambda, C^{g} A^{h} \mu\right)$. Then by $(4.5 a)$

$$
\begin{aligned}
S_{\rho \lambda}^{(k)} S_{(1,2) \mu}^{(k+1)} & =S_{\rho, C^{e} A^{f} \lambda}^{(k)} S_{(1,2), C^{g} A^{h} \mu}^{(k+1)}=S_{\rho \lambda}^{(k)} S_{(1,2), C^{g} A^{h} \mu}^{(k+1)}, \\
S_{(1,2), \lambda}^{(k)} S_{\rho \mu}^{(k+1)} & =S_{(1,2), C^{e} A^{f} \lambda}^{(k)} S_{\rho_{,} C^{g} A^{h} \mu}^{(k+1)}=S_{(1,2), C^{e} A^{f} \lambda}^{(k)} S_{\rho \mu}^{(k+1)} .
\end{aligned}
$$

But Lemma 3(b) and the fact that $S_{\rho \nu}^{(\ell)} \neq 0$ gives us $\mu=C^{g} A^{h} \mu, \lambda=C^{e} A^{f} \lambda-$ i.e. $\sigma^{\prime \prime}(\lambda \mu)=(\lambda \mu)$. Thus $M^{\prime}=M^{\sigma^{\prime}}$. QED

Thus, for each $k$ there will be 24 or 16 automorphism invariants. Of these, the only ones satisfying $(2.11 c)$ (so that they correspond to coset invariants) are:

$$
\begin{aligned}
& k \equiv 0(\bmod 3):(0,0,0,0),(0,0,0,1)^{c},{ }^{c}(1,1,-1,0),{ }^{c}(1,1,1,1)^{c} ; \\
& k \equiv 1(\bmod 3):(0,0,0,0),(0,0,0,-1)^{c},{ }^{c}(1,0,0,0),{ }^{c}(1,0,0,-1)^{c} ; \\
& k \equiv 2(\bmod 3):(0,0,0,0),(0,1,-1,-1)^{c},{ }^{c}(-1,0,0,0),{ }^{c}(-1,-1,-1,-1)^{c} .
\end{aligned}
$$

\section{The physical invariants of $s u(3)_{k} \oplus s u(3)_{k+1}$ involving chiral extensions}

In this section we complete the proof of Thm.2. We will begin by finding all possibilities for the maximally extended chiral algebras, and then find all automorphisms of those chiral algebras.

\subsection{The weights which can couple to $(\rho \rho)$}

In this subsection we begin the search for all possible chiral algebra extensions by finding the possible weights $(\lambda, \mu) \in \mathcal{P}(k)$ which can couple to $(\rho \rho)$, i.e. those $(\lambda, \mu)$ which satisfy the norm condition ( $T$-invariance)

$$
\frac{2}{k^{\prime}}+\frac{2}{k^{\prime}+1} \equiv \frac{\lambda^{2}}{k^{\prime}}+\frac{\mu^{2}}{k^{\prime}+1}(\bmod 2)
$$


as well as satisfy the parity rules (4.3) (with $\kappa=\nu=\rho$ ). Any weights $(\lambda, \mu$ ) for which $M_{\rho \rho, \lambda \mu} \neq 0$ for some physical invariant $M$, must necessarily satisfy (5.1a) and (4.3). We will also impose the condition $(2.11 a)$, namely that

$$
t(\lambda)+t(\mu) \equiv 0(\bmod 3)
$$

For arbitrary algebras, this step (i.e. enumerating the possible rho-couplings) is quite tedious. Fortunately the hard work has already been done by [8], and all we have to do is collect the pieces. Let us begin by reviewing some observations from [10].

For a given physical invariant $M$ of $s u(3)_{k} \oplus s u(3)_{k+1}$, write $\mathcal{P}_{L}=\left\{(\lambda, \mu) \in \mathcal{P}(k) \mid M_{\lambda \mu, \kappa \nu}\right.$ $\neq 0$ for some $\kappa, \nu\}$, and $\mathcal{J}_{L}=\left\{A_{k}^{a} A_{k+1}^{b} \mid M_{A^{a} \rho A^{b} \rho, \rho \rho} \neq 0\right\}$. $\mathcal{P}_{R}$ and $\mathcal{J}_{R}$ can be defined similarly. $\mathcal{J}_{L}(\lambda \mu)$ denotes the orbit, and $\left\|\mathcal{J}_{L}(\lambda \mu)\right\|$ its cardinality. Then we have

Lemma 4. (a) For each $(\lambda, \mu) \in \mathcal{P}(k)$, define $s(\lambda, \mu)=\sum_{\kappa, \nu} M_{\rho \rho, \kappa \nu} S_{\lambda \kappa}^{(k)} S_{\mu \nu}^{(k+1)}$. Then each $s(\lambda, \mu) \geq 0$, and $s(\lambda, \mu)>0$ iff $(\lambda, \mu) \in \mathcal{P}_{L}$.

(b) For any $a, b, c, d, M_{A^{a} \rho A^{b} \rho, A^{c} \rho A^{d} \rho}=1$ holds iff $M_{A^{a} \lambda A^{b} \mu, A^{c} \nu A^{d} \kappa}=M_{\lambda \mu, \nu \kappa}$ for all $\lambda, \mu, \nu, \kappa$, iff $a t(\lambda)+b t(\mu) \equiv \operatorname{ct}(\nu)+d t(\kappa)(\bmod 3)$ whenever $M_{\lambda \mu, \nu \kappa} \neq 0$. In particular, $\forall A_{k}^{a} A_{k+1}^{b} \in \mathcal{J}_{L}, M_{A^{a} \lambda A^{b} \mu, \kappa \nu}=M_{\lambda \mu, \kappa \nu}$.

The same comments apply to $\mathcal{P}_{R}$ and $\mathcal{J}_{R}$, of course. Lemma 4 holds for general $g$, and more generally, any rational conformal field theory with a centre. It is proven in [7] (see also Lemma 1 in [10]). (a) follows from $S^{(k, k+1)} M=M S^{(k, k+1)}$ and the fact that all $S_{\rho \rho, \kappa \nu}$ are positive. (b) follows from calculations from $M=S^{(k, k+1) \dagger} M S^{(k, k+1)}$, along the lines of how (2.8c) implied (2.8a) and (2.8b). Note that (b) implies $\mathcal{J}_{L, R}$ are (Abelian) groups.

A final useful result concerns the eigenvalues of $M$. Write $M$ as the direct sum

$$
M=\oplus_{i} M_{i}=\left(\begin{array}{cccc}
M_{1} & 0 & \cdots & 0 \\
0 & M_{2} & & 0 \\
\vdots & & \ddots & \\
0 & 0 & & M_{\alpha}
\end{array}\right)
$$

of indecomposable submatrices $M_{i}$. Each weight $(\lambda, \mu) \in \mathcal{P}(k)$ will be 'contained' in one and only one $M_{i}$. For convenience always choose $M_{1}$ to contain $(\rho \rho)$. Because every entry of each $M_{i}$ is non-negative, then $M_{i}$ has a non-negative eigenvalue $r\left(M_{i}\right)$, called the Perron-Frobenius eigenvalue, with many remarkable properties. The most important is that if $s$ is any other (possibly complex) eigenvalue of $M_{i}$ then $|s| \leq r\left(M_{i}\right)$. See e.g. [10] for more details. These $M_{i}$ will usually turn out to be an $m \times m$ matrix of the form

$$
B_{(\ell, m)}=\left(\begin{array}{ccc}
\ell & \cdots & \ell \\
\vdots & & \vdots \\
\ell & \cdots & \ell
\end{array}\right),
$$

for some $\ell, m$. It is easy to see that $r\left(B_{(\ell, m)}\right)=\ell m$.

Define sets $\mathcal{R}_{L}$ and $\mathcal{R}_{R}$ by $(\lambda, \mu) \in \mathcal{R}_{L}$ iff $M_{\lambda \mu, \rho \rho} \neq 0$, and $(\lambda, \mu) \in \mathcal{R}_{R}$ iff $M_{\rho \rho, \lambda \mu} \neq 0$. The following result comes from Lemma 3 in [10]. 
Lemma 5. (a) Suppose $M$ has $M_{1}=B_{(1, m)}$ for some $m$. Then for each $i$, either $M_{i}=(0)$ or $r\left(M_{i}\right)=m$. Also, for each $(\lambda, \mu) \in \mathcal{P}(k), \sum_{\kappa \nu} M_{\lambda \mu, \kappa \nu}^{2} \leq m^{2} /\left\|\mathcal{J}_{L}(\lambda \mu)\right\|$.

(b) Now suppose $\mathcal{R}_{L}=\mathcal{J}_{L}(\rho \rho)$ and $\mathcal{R}_{R}=\mathcal{J}_{R}(\rho \rho)$. Suppose $M_{\lambda \mu, \kappa \nu} \neq 0$. Then $M_{\lambda \mu, \kappa \nu} \leq$ $\left\|\mathcal{J}_{L}\right\| / \sqrt{\left\|\mathcal{J}_{L}(\lambda \mu)\right\|\left\|\mathcal{J}_{R}(\kappa \nu)\right\|}$. If in addition $(\lambda, \mu)$ is not a fixed point of $\mathcal{J}_{L}$ (i.e. $J \in$ $\mathcal{J}_{L}, J \neq 0$, implies $J(\lambda, \mu) \neq(\lambda, \mu)$ ) and also $(\kappa \nu)$ is not a fixed point of $\mathcal{J}_{R}$, then $M_{\lambda \mu, \kappa \nu}=1$; moreover, $M_{\lambda \mu, \alpha \beta} \neq 0$ iff $(\alpha \beta) \in \mathcal{J}_{R}(\kappa \nu)$, and $M_{\alpha \beta, \kappa \nu} \neq 0$ iff $(\alpha \beta) \in$ $\mathcal{J}_{L}(\lambda, \mu)$.

We are now ready for the main result of this subsection:

Proposition 1. Let $M$ be a physical invariant of $s u(3)_{k} \oplus s u(3)_{k+1}$, satisfying (2.11), so that a coset invariant can be obtained from it. Then $M_{\rho \rho, \lambda \mu}=M_{\lambda \mu, \rho \rho} \in\{0,1\}$, for all $(\lambda, \mu) \in \mathcal{P}(k)$. Thus $\mathcal{R}_{L}=\mathcal{R}_{R}$ and $\mathcal{J}_{L}=\mathcal{J}_{R}$. Putting $\rho^{\prime}=\left(\frac{k+1}{2}, \frac{k+1}{2}\right), \rho^{\prime \prime}=\left(\frac{k+2}{2}, \frac{k+2}{2}\right)$, the possibilities for $\mathcal{R}_{L}$ are given by:

(i) $k \equiv 0(\bmod 3), k \neq 9,21$ : either $\mathcal{R}_{L}=\{(\rho \rho)\}$ or $\mathcal{R}_{L}=\left\{(\rho \rho),(A \rho, \rho),\left(A^{2} \rho, \rho\right)\right\}$;

(ii) $k \equiv 1(\bmod 3), k \neq 4$ : either $\mathcal{R}_{L}=\{(\rho, \rho)\}$ or $\mathcal{R}_{L}=\left\{(\rho, \rho),(A \rho, A \rho),\left(A^{2} \rho, A^{2} \rho\right)\right\}$;

(iii) $k \equiv 2(\bmod 3), k \neq 5,8,20$ : either $\mathcal{R}_{L}=\{(\rho, \rho)\}$ or $\mathcal{R}_{L}=\left\{(\rho, \rho),(\rho, A \rho),\left(\rho, A^{2} \rho\right)\right\}$;

(iv) $k=4: \mathcal{R}_{L}$ is either given in (ii), or equals $\left\{(\rho \rho),\left(\rho \rho^{\prime \prime}\right)\right\}$ or $\left\{\left(A^{a} \rho, A^{a} \rho\right),\left(A^{a} \rho, A^{a} \rho^{\prime \prime}\right)\right.$ $\mid a=0,1,2\}$;

$k=5: \mathcal{R}_{L}$ is either given in (iii), or equals $\left\{(\rho \rho),\left(\rho^{\prime} \rho\right)\right\}$ or $\left\{\left(\rho, A^{a} \rho\right),\left(\rho^{\prime}, A^{a} \rho\right) \mid a=\right.$ $0,1,2\}$;

$k=8: \mathcal{R}_{L}$ is either given in (iii), or equals $\left\{\left(\rho, A^{a} \rho\right),\left(\rho, A^{a} \rho^{\prime \prime}\right) \mid a=0,1,2\right\}$;

$k=9: \mathcal{R}_{L}$ is either given in (i), or equals $\left\{\left(A^{a} \rho, \rho\right),\left(A^{a} \rho^{\prime}, \rho\right) \mid a=0,1,2\right\}$;

$k=20: \mathcal{R}_{L}$ is either given in (iii), or equals

$$
\left\{\left(\rho, A^{a} \rho\right),\left(\rho, A^{a} \rho^{\prime \prime}\right),\left(\rho, A^{a}(5,5),\left(\rho, A^{a}(7,7)\right) \mid a=0,1,2\right\}\right.
$$

$k=21: \mathcal{R}_{L}$ is either given in (i), or equals

$$
\left\{\left(A^{a} \rho, \rho\right),\left(A^{a} \rho^{\prime}, \rho\right),\left(A^{a}(5,5), \rho\right),\left(A^{a}(7,7), \rho\right) \mid a=0,1,2\right\} .
$$

Proof Consider first $k \equiv 0(\bmod 12)$. We see from Lemma 2 that $(\lambda, \mu) \in \mathcal{R}_{L}$ means $\lambda=A^{a} \rho$ and $\mu=A^{b} \rho$ or $\mu=A^{b} \rho^{\prime \prime}$, for some $a, b=0,1,2$. Equation (5.1b) forces $b=0$. Put $m_{L}=\sum_{a=0}^{2} M_{A^{a} \rho \rho, \rho \rho}, m_{L}^{\prime}=\sum_{a=0}^{2} M_{A^{a} \rho \rho^{\prime \prime}, \rho \rho}$.

Now put $\lambda=\rho, \mu=(1,4)$ in Lemma 4(a):

$$
\left(m_{L}+m_{L}^{\prime}\right) \sin \left[\frac{2 \pi}{k^{\prime}+1}\right]+\left(m_{L}-m_{L}^{\prime}\right) \sin \left[\frac{8 \pi}{k^{\prime}+1}\right]-\left(m_{L}+m_{L}^{\prime}\right) \sin \left[\frac{10 \pi}{k^{\prime}+1}\right] \geq 0 .
$$

From Lemma $4(\mathrm{~b})$ and $M_{\rho \rho, \rho \rho}=1$ we know $m_{L}=1$ or $3: m_{L}=1$ corresponds to $\mathcal{J}_{L}=\left\{A_{k}^{0}\right\} ; m_{L}=3$ corresponds to $\mathcal{J}_{L}=\left\{A_{k}^{0}, A_{k}, A_{k}^{2}\right\}$. In either case, (5.4) forces $m_{L}>m_{L}^{\prime}($ since $k>9)$, while Lemma $4(\mathrm{~b})$ implies that if $m_{L}^{\prime} \neq 0$ then $m_{L} \leq m_{L}^{\prime}$. Together these tell us that $m_{L}^{\prime}=0$.

Similar arguments apply to $m_{R}$ and $m_{R}^{\prime}$. That we have $\mathcal{R}_{L}=\mathcal{R}_{R}$ here, follows from $S^{(k, k+1)} M=M S^{(k, k+1)}$ evaluated at $(\rho \rho, \rho \rho)$ : it says $m_{L}=m_{R}$.

The other $k$ are all handled similarly. (See especially Claim 7 in [10].) QED 


\subsection{Simple current chiral extensions and their automorphisms}

Write $\mathcal{R}$ for $\mathcal{R}_{L}=\mathcal{R}_{R}$, and $\mathcal{J}$ for $\mathcal{J}_{L}=\mathcal{J}_{R}$. In this subsection we find all $M$ for which $\mathcal{R} \subseteq\left(\mathcal{O}_{0}^{k} \rho, \mathcal{O}_{0}^{k+1} \rho\right)$. We see from Prop. 1 that indeed all $M$ satisfy that condition, except at the six exceptional levels $k=4,5,8,9,20,21$.

Note that either $\|\mathcal{J}\|=1$ or 3 . If it equals 1 , then $M$ must be an automorphism invariant (see Thm. 3 in [6]), hence is listed at the end of Sect.4. The argument for $\|\mathcal{J}\|=3$ will closely follow that of Sect.4.

Consider first the easiest case: $k \equiv 1(\bmod 3)(k \neq 4)$. Then $\mathcal{J}=\left\{J^{0}, J^{1}, J^{2}\right\}$, where $J=A_{k} A_{k+1}$. Lemma 4(b) tells us that $\mathcal{P}:=\mathcal{P}_{L}=\mathcal{P}_{R}=\{(\lambda \mu) \in \mathcal{P}(k) \mid t(\lambda) \equiv$ $-t(\mu)(\bmod 3)\}$. For each $(\lambda, \mu) \in \mathcal{P}$, define the $J$-orbit $\langle\lambda \mu\rangle=\left\{(\lambda, \mu), J(\lambda, \mu), J^{2}(\lambda, \mu)\right\}$, and

$$
c h_{\langle\lambda \mu\rangle}=\sum_{\left(\lambda^{\prime} \mu^{\prime}\right) \in\langle\lambda \mu\rangle} \chi_{\lambda^{\prime}}^{(k)} \chi_{\mu^{\prime}}^{(k+1)} .
$$

The special thing here is that there are no fixed points of $\mathcal{J}$. So Lemma $5(\mathrm{~b})$ tells us that there exists a permutation $\sigma$ of the $J$-orbits $\langle\lambda \mu\rangle \subset \mathcal{P}$ such that the partition function $Z$ associated to $M$ can be written

$$
Z=\sum_{\langle\lambda \mu\rangle} c h_{\langle\lambda \mu\rangle} c h_{\sigma\langle\lambda \mu\rangle}^{*}
$$

So our task reduces to finding all bijections $\sigma$ such that $(5.5 b)$ is a modular invariant. Define a matrix $S^{e}$ by

$$
S_{\langle\lambda \mu\rangle,\langle\kappa \nu\rangle}^{e}:=3 S_{\lambda \kappa}^{(k)} S_{\mu \nu}^{(k+1)} .
$$

Then $S^{e}$ is unitary and symmetric, and $M$ commutes with $S^{(k, k+1)}$ iff $\sigma$ is a symmetry of $S^{e}$. We may formally define "fusion rules" by Verlinde's formula:

$$
N_{\langle\lambda \mu\rangle,\langle\lambda \mu\rangle,\langle\lambda \mu\rangle}^{e}:=\sum_{\langle\kappa \nu\rangle} \frac{\left(S_{\langle\lambda \mu\rangle,\langle\kappa \nu\rangle}^{e}\right)^{3}}{S_{\langle\rho \rho\rangle,\langle\kappa \nu\rangle}^{e}}=\sum_{\langle\kappa \nu\rangle} \sum_{a, b=0}^{2} \frac{\left(S_{\lambda, A^{a} \kappa}^{(k)}\right)^{3}\left(S_{\mu, A^{b} \nu}^{(k+1)}\right)^{3}}{S_{\rho, A^{a} \kappa}^{(k)} S_{\rho, A^{b} \nu}^{(k+1)}}=N_{\lambda \lambda \lambda}^{(k)} N_{\mu \mu \mu}^{(k+1)},
$$

where the second equality arises by using (5.6a) and (2.2c) (the cube cancels the extra phases which appear). $\sigma$ will also be a symmetry of these $N^{e}$.

Either $k^{\prime}$ or $k^{\prime}+1$ will be odd - that one will be coprime to 6 for $k \equiv 1(\bmod 3)$. Say $k^{\prime}$ is odd. Write $\sigma\langle\lambda \mu\rangle=\left\langle\lambda^{\prime} \mu^{\prime}\right\rangle$. Then Lemma 1 says $\lambda^{\prime} \in \mathcal{O} \lambda$. As in the proof of Claim 3 , eqs.(5.6) imply eqs.(4.7), so Lemma $3\left(\right.$ a) gives us $\mu^{\prime} \in \mathcal{O} \mu$.

Now $C \rho=\rho$, so $\sigma\langle\rho(1,4)\rangle$ must equal $\left\langle\rho, C^{a} A^{b}(1,4)\right\rangle$ for some $a, b$; but $t\left(C^{a} A^{b}(1,4)\right) \equiv$ $-t(\rho)(\bmod 3)$, so $b=0$. Conjugating if necessary (see eq.(4.10a)), we may suppose $\sigma\langle\rho(1,4)\rangle=\langle\rho(1,4)\rangle$. Similarly, conjugating if necessary, we may suppose $\sigma\langle(1,4) \rho\rangle=$ $\langle(1,4) \rho\rangle$. Write $\sigma\langle\lambda \mu\rangle=\left\langle C^{w} A^{x} \lambda, C^{y} A^{z} \mu\right\rangle ;(5.6 a)$ gives us $S_{(14), \mu}^{(k+1)}=S_{(14), C^{y} A^{z} \mu}^{(k+1)}, S_{(14), \lambda}^{(k)}=$ $S_{(14), C^{w} A^{x} \lambda}^{(k)}$ Lemma 3(c) then requires $\mu=A^{i} \mu^{\prime}$ and $\lambda=A^{j} \lambda^{\prime}$ for some $i, j . t(\lambda)+t(\mu) \equiv$ $t\left(\lambda^{\prime}\right)+t\left(\mu^{\prime}\right) \equiv 0(\bmod 3)$ then forces $i=j$, so $\sigma\langle\lambda \mu\rangle=\langle\lambda \mu\rangle$.

The final result (reintroducing the conjugations and ensuring $\sigma\langle\lambda \mu\rangle \in \mathcal{P}$ ) is that either $\sigma\langle\lambda \mu\rangle=\langle\lambda \mu\rangle \forall\langle\lambda \mu\rangle$, or $\sigma\langle\lambda \mu\rangle=\langle C \lambda, C \mu\rangle \forall\langle\lambda \mu\rangle$. 
The arguments for $k \equiv 0$ and $k \equiv 2(\bmod 3)(k \neq 5,8,9,20,21)$ are identical to each other, and similar to $k \equiv 1(\bmod 3)$ except for the presence of the fixed points $\left(\left(k^{\prime} / 3, k^{\prime} / 3\right), \mu\right)$ and $\left(\lambda,\left(\left[k^{\prime}+1\right] / 3,\left[k^{\prime}+1\right] / 3\right)\right)$, respectively. Consider the case $k \equiv 0(\bmod 3)$. Here, $\mathcal{P}:=\mathcal{P}_{L}=\mathcal{P}_{R}=\{(\lambda, \mu) \in \mathcal{P}(k) \mid t(\lambda) \equiv 0(\bmod 3)\}$. Write $f=\left(k^{\prime} / 3, k^{\prime} / 3\right)$, $\langle\lambda\rangle=\left\{\lambda, A_{k} \lambda, A_{k}^{2} \lambda\right\}$,

$$
\operatorname{ch}_{\langle\lambda\rangle \mu}=\sum_{\lambda^{\prime} \in\langle\lambda\rangle} \chi_{\lambda^{\prime}}^{(k)} \chi_{\mu}^{(k+1)} .
$$

Then as before,

$$
Z=\sum M_{\langle\lambda\rangle \mu,\langle\kappa\rangle \nu}^{e} \operatorname{ch}_{\langle\lambda\rangle \mu} \operatorname{ch}_{\langle\kappa\rangle \nu}^{*} .
$$

Choose any $(\lambda, \mu) \in \mathcal{P}, \lambda \neq f$, such that $M_{\lambda \mu, f \nu}=0$ for all $\nu$. Then Lemma 5(b) says there exists a map $\sigma$ such that

$$
M_{\langle\lambda\rangle \mu,\langle\kappa\rangle \nu}^{e}=\delta_{\sigma(\langle\lambda\rangle \mu),\langle\kappa\rangle \nu} .
$$

Suppose we have $\sigma(\langle\lambda\rangle \mu)=\left\langle\lambda^{\prime}\right\rangle \mu^{\prime}$ and $\sigma(\langle\kappa\rangle \nu)=\left\langle\kappa^{\prime}\right\rangle \nu^{\prime}$. Look at $S^{(k, k+1)} M=M S^{(k, k+1)}$, we get an equation resembling (4.5):

$$
S_{\lambda \kappa}^{(k)} S_{\mu \nu}^{(k+1)}=S_{\lambda^{\prime} \kappa^{\prime}}^{(k)} S_{\mu^{\prime} \nu^{\prime}}^{(k+1)} .
$$

By Lemma 2, $M_{\rho \mu, f \nu}=0$ for all $\mu, \nu$, so we may write $\sigma(\langle\rho\rangle \mu)=\left\langle\lambda^{\prime}\right\rangle \mu^{\prime}$. Lemma 2 tells us that $\lambda^{\prime} \in\langle\rho\rangle$ or $\left\langle\rho^{\prime}=\left(\frac{k+1}{2}, \frac{k+1}{2}\right)\right\rangle$ (when $k=9,15,21,57$ we have additional possibilities, but they all succumb to similar arguments).

Suppose for contradiction that $\lambda^{\prime}=\rho^{\prime}$. Apply (5.8b) with $\nu=\kappa=\rho$ : we find (see (4.6c)) that $S_{\rho \rho^{\prime}}^{(k)} / S_{\rho \rho}^{(k)}$ must be a rational number. But we proved in Claim 3 that this could only happen for $k^{\prime} \leq 6$, yet Lemma 2 and $k \equiv 0(\bmod 3)$ tells us $k^{\prime} \geq 12$. Therefore $\sigma(\langle\rho\rangle \mu)=\langle\rho\rangle \mu^{\prime}$.

(5.8b) now tells us (choosing $\lambda=\kappa=\rho$ ) that $S_{\mu \nu}^{(k+1)}=S_{\mu^{\prime} \nu^{\prime}}^{(k+1)}$. Thus the map $\mu \mapsto \mu^{\prime}$ defines an automorphism invariant of $s u(3)_{k+1}$, so equals $\overline{\mathcal{A}}_{k+1}$ or $\overline{\mathcal{D}}_{k+1}$ or their conjugations. Multiplying $M$ by ( $\overline{\mathcal{A}}_{k} \otimes$ this automorphism invariant) allows us to suppose that $\sigma(\langle\rho\rangle \mu)=\langle\rho\rangle \mu$, for all $\mu$.

Now choose any $\lambda$ with $t(\lambda) \equiv 0$. Then

$$
\sum_{\alpha, \beta} S_{\rho \alpha}^{(k)} S_{\nu \beta}^{(k+1)} M_{\alpha \beta, \lambda \mu}=\sum_{\alpha, \beta} M_{\rho \nu, \alpha \beta} S_{\alpha \lambda}^{(k)} S_{\beta \mu}^{(k+1)}=3 S_{\rho \lambda}^{(k)} S_{\nu \mu}^{(k+1)}
$$

holds for any $\nu$. Multiplying this by $S_{\nu \kappa}^{(k+1) *}$ for any $\kappa$ and summing over $\nu$, we have

$$
\sum_{\alpha} S_{\rho \alpha}^{(k)} M_{\alpha \kappa, \lambda \mu}=3 S_{\rho \lambda}^{(k)} \delta_{\kappa \mu} .
$$

By positivity, we see from this that $M_{\alpha \beta, \lambda \mu} \neq 0$ requires $\beta=\mu$.

The remainder of the argument is as in Thm.3 of [10]. Namely, $M_{(2,2) \rho, f \mu}=0$ for all $\mu$ (unless $k=3$, which can be worked out by $T$-invariance, and $k=9$, which gives 
us the exceptional $\left.\overline{\mathcal{E}}_{9}^{(2)} \otimes \overline{\mathcal{A}}_{10}\right)$; from this we get $\sigma(\langle 2,2\rangle \rho)=\langle 2,2\rangle \rho ; M_{f \mu, \lambda \mu}=0$ for any $\lambda \neq f$ because otherwise $S^{(k, k+1)} M=M S^{(k, k+1)}$ evaluated at $(\rho \rho, \lambda \mu)$ and at $((2,2) \rho, \lambda \mu)$ yield two incompatible equations. Therefore the fixed point behaviour is trivial: $M_{f \mu, \lambda \nu}=$ $3 \delta_{f \lambda} \delta_{\mu \nu} . \sigma$ can now be extended to all $\langle\lambda\rangle \mu$ by defining $\sigma(\langle f\rangle \mu)=\langle f\rangle \mu$. Then (5.8b) holds $\forall \lambda, \mu, \nu, \kappa \in \mathcal{P}$. Looking at $N_{\lambda f f}^{(k)} N_{\mu \mu \mu}^{(k+1)}$, and using Lemma 3(c), completes the argument.

The final result, reintroducing the automorphisms and imposing $(2.11 c)$, is that $M=$ $\mathcal{M}_{k} \otimes \mathcal{M}_{k+1}$ where $\mathcal{M}_{k}=\overline{\mathcal{D}}_{k}$ or $\overline{\mathcal{D}}_{k}^{c}$, and $\mathcal{M}_{k+1}=\overline{\mathcal{A}}_{k+1}$ or $\overline{\mathcal{D}}_{k+1}^{c}$. Thus together with Prop.1 and Claim 4, we have proven Thm.2 for all $k$, except for $k=4,5,8,9,20$ and 21.

\subsection{The exceptional levels}

In this subsection we complete the proof of Thm.2 by considering the remaining levels $k=4,5,8,9,20,21$. We know the values of $M_{\lambda \mu, \rho \rho}$ and $M_{\rho \rho, \lambda \mu}$ for these levels (Prop.1); the task is to go from these to all $M_{\lambda \mu, \kappa \nu}$. We have many tools for doing this: most notably Lemmas 1,2,4 and 5, as well as direct use of $T$-invariance and the relation $M S^{(k, k+1)}=$ $S^{(k, k+1)} M$. The arguments for each level are all essentially the same; we will explicitly give the one for $k=4$. See Thm.3 of [10] for more details.

Let us begin with $k=4$, and $\mathcal{R}=\left\{(\rho, \rho),\left(\rho, \rho^{\prime \prime}\right)\right\}$. Then by Prop.1, $M_{\rho \rho, \lambda \mu}=$ $M_{\lambda \mu, \rho \rho}=0$, for all $(\lambda, \mu) \in \mathcal{P}(k)$, except for $M_{\rho \rho, \rho \rho}=M_{\rho \rho, \rho \rho^{\prime \prime}}=M_{\rho \rho^{\prime \prime}, \rho \rho}=1$. Now suppose $M_{\lambda \mu, \rho \rho^{\prime \prime}} \neq 0$. Choose $\ell$ coprime to $3 \cdot 7 \cdot 8$, such that $\ell \equiv \pm 1(\bmod 3 \cdot 7)$ and $\ell \equiv 5(\bmod 3 \cdot 8)-$ e.g. $\ell=125$. Then $[\ell \rho]_{4}=\rho,\left[\ell \rho^{\prime \prime}\right]_{5}=\rho$, so by the parity rule $(4.2 a)$ we get $M_{\lambda^{\prime} \mu^{\prime}, \rho \rho}=M_{\lambda \mu, \rho \rho^{\prime \prime}} \neq 0$, where $\lambda^{\prime}=[\ell \lambda]_{4}$ and $\mu^{\prime}=[\ell \mu]_{5}$. Thus $M_{\lambda \mu, \rho \rho^{\prime \prime}}=1$, and $\left(\lambda^{\prime} \mu^{\prime}\right)=(\rho \rho)$ or $\left(\rho \rho^{\prime \prime}\right)$, i.e. $(\lambda \mu)=\left(\rho \rho^{\prime \prime}\right)$ or $(\rho \rho)$.

What we have shown is that $M_{1}=B_{(1,2)}$ (see (5.3)). By Lemma 4(a), $\mathcal{P}=P_{++}(s u(3), 4) \times$ $\left(\mathcal{O}_{0} \rho \cup \mathcal{O}_{0}(3,3) \cup \mathcal{O}(1,3)\right)$. Equations (4.1) and (4.3) tell us $M_{\lambda \mu, \kappa \nu} \neq 0$ requires $\kappa \in \mathcal{O} \lambda$ and either $\mu, \nu \in \mathcal{O}_{0} \rho \cup \mathcal{O}_{0}(3,3)$, or $\mu, \nu \in \mathcal{O}(1,3)$. Now, computing $\left(S^{(k, k+1)} M\right)_{\lambda \mu, \rho \rho}=$ $\left(M S^{(k, k+1)}\right)_{\lambda \mu, \rho \rho}$ for any $(\lambda, \mu) \in \mathcal{P}$ gives us $\sum_{\kappa \nu} M_{\lambda \mu, \kappa \nu}=2$, and if $\mu \in \mathcal{O}_{0} \rho \cup \mathcal{O}_{0}(3,3)$ we get further that there exist weights $\left(\lambda^{\prime} \mu^{\prime}\right),\left(\lambda^{\prime \prime} \mu^{\prime \prime}\right) \in \mathcal{P}$ such that $\mu^{\prime} \in \mathcal{O}_{0} \rho$ and $\mu^{\prime \prime} \in \mathcal{O}_{0}(3,3)$, and $M_{\lambda \mu, \lambda^{\prime} \mu^{\prime}}=M_{\lambda \mu, \lambda^{\prime \prime} \mu^{\prime \prime}}=1$.

For each $a$, there exist $b, c$ such that $M_{\rho A^{a} \rho, A^{b} \rho A^{c} \rho} \neq 0$. Then $(2.11 a)$ and $T$-invariance force $b=0$ and $a=c$. Thus by Lemma 4(b) and (2.11b), $M_{\lambda \mu, \kappa \nu}=M_{A^{a} \lambda A^{b} \mu, A^{a} \kappa A^{b} \nu}$, and $M_{\lambda \mu, \kappa \nu} \neq 0$ implies $t(\lambda) \equiv t(\kappa)$ and $t(\mu) \equiv t(\nu)$.

We can now completely determine $M$, once we know the values of $M_{\rho(1,3), \rho(1,3)}$, $M_{(1,2) \rho,(1,2) \rho}, M_{(1,2) \rho,(1,2)(3,3)}, M_{(1,2)(1,3),(1,2)(1,3)}$ and $M_{(1,2)(1,3),(1,2)(4,3)}$. By multiplying $M$ by $\overline{\mathcal{D}}_{4}^{c} \otimes \overline{\mathcal{A}}_{5}$ if necessary, we can force $M_{(1,2) \rho,(1,2) \rho}=1$. The relation $S^{(k, k+1)} M=$ $M S^{(k, k+1)}$ evaluated at certain weights now forces $M=\overline{\mathcal{A}}_{4} \otimes \overline{\mathcal{E}}_{5}$.

The other anomolous possibility for $\mathcal{R}$ when $k=4$ is $\mathcal{R}=\left\{A^{a}(\rho, \rho), A^{a}\left(\rho, \rho^{\prime \prime}\right) \mid a=\right.$ $0,1,2\}$. Here $\mathcal{J}$ has order 3 and $M_{1}=B_{(1,6)}$, but otherwise the argument is very similar to the one just given.

\section{Conclusion}

Our main results are already pointed out in the Introduction. We conclude with a brief discussion of possible future directions of research. 
The correspondence of section 2 , between the physical modular invariants of diagonal coset theories and WZNW tensor products, was the starting point of this work. We restricted attention here to diagonal cosets without fixed points, because of the simplicity of their field identifications. The switch of weights should also yield a correspondence for more general classes of GKO cosets. We hope to address this in later work. The correspondence might be helpful in calculating other quantities of interest in coset theories, such as correlation functions.

The ultimate goal of any modular invariant classification such as ours is a deeper understanding of (rational) conformal field theories in general. There are two ways progress may be made. First, the truths used to complete a particular classification can be shown valid in more general contexts. Since coset theories comprise (at least) a large part of all rational conformal theories, it would be worthwhile to try to prove some of our results for an arbitrary rational theory, perhaps with a centre. Second, a pattern may emerge in the completed classifications that can be extended to other theories. Presumably, such a pattern would be some generalisation of the famous A-D-E results of [4]. Perhaps the connection with [16] points the way.

\section{Acknowledgements}

T.G. would like to thank Antoine Coste, Quang Ho-Kim, Philippe Ruelle, and Yassen Stanev for valuable communications, and the IHES for its generosity. M.W. thanks Pierre Mathieu for helpful discussions.

\section{References}

1. Bais, F. A., Bouwknegt, P., Surridge, M., Schoutens, K.: Coset construction for extended Virasoro algebras. Nucl. Phys. B304 371-391 (1988);

Di Francesco, P., Zuber, J.-B.: SU(N) lattice integrable models and modular invariants. In: Proceedings of Trieste conference on Recent Developments in Conformal Field Theories (1989)

2. Bégin, L., Mathieu, P., Walton, M. A.: siu $(3)_{k}$ fusion coefficients. Mod. Phys. Lett. A7 3255-3265 (1992)

3. Bernard, D.: String characters from Kac-Moody automorphisms. Nucl. Phys. B288 628-648 (1987);

Altschuler, D., Lacki, J., Zaugg, P.: The affine Weyl group and modular invariant partition functions. Phys. Lett. 205B 281-284 (1988);

Felder, G., Gawedzki, K., Kupiainen, A.: Spectra of Wess-Zumino-Witten models with arbitrary simple groups. Commun. Math. Phys. 117 127-158 (1988);

Ahn, C., Walton, M. A.: Spectra of strings on nonsimply-connected group manifolds. Phys. Lett. 223B 343-348 (1989)

4. Cappelli, A., Itzykson, C., Zuber, J.-B.: The A-D-E classification of $A^{(1)}$ and minimal conformal field theories. Commun. Math. Phys. 113 1-26 (1987); Modular invariant partition functions in two dimensions. Nucl. Phys. B280 [FS18] 445-465 (1987) 
5. Christe, P., Ravanani, F.: $G_{N} \otimes G_{L} / G_{N+L}$ conformal field theories and their modular invariant partition functions. Int. J. Mod. Phys. A4 897-920 (1989)

6. Gannon, T.: WZW commutants, lattices, and level-one partition functions. Nucl. Phys. B396 708-736 (1993)

7. Gannon, T.: Towards a classification of $\mathrm{su}(2) \oplus \cdots \oplus \mathrm{su}(2)$ modular invariant partition functions. IHES preprint P/94/21 (hep-th/9402074)

8. Gannon, T.: The classification of affine $\mathrm{SU}(3)$ modular invariant partition functions. Commun. Math. Phys. 161 233-264 (1994)

9. Gannon, T., Ho-Kim, Q.: The low level modular invariant partition functions of ranktwo algebras. Int. J. Mod. Phys. (in press) (hep-th/9304106); The rank four heterotic modular invariant partition functions. Nucl. Phys. B (in press) (hep-th/9402027)

10. Gannon, T.: The classification of SU(3) modular invariants revisited. IHES preprint (hep-th/9404185)

11. Goddard, P., Kent, A., Olive, D.: Virasoro algebras and coset space models. Phys. Lett. 152B 88-92 (1985); Unitary representations of the Virasoro and Super-Virasoro algebras. Commun. Math. Phys. 103 105-119 (1986)

12. Intriligator, K.: Bonus symmetry in conformal field theory. Nucl. Phys. B332 541-565 (1990);

Schellekens, A. N., Yankielowicz, S.: Simple currents, modular invariants and fixed points. Int. J. Mod. Phys. 5A 2903-2952 (1990)

13. Kac, V. G.: Infinite dimensional Lie algebras, 3rd ed. Cambridge: Cambridge University Press 1990

14. Kac, V., Peterson, D.: Infinite dimensional lie algebras, theta functions, and modular forms. Adv. Math. 53 125-264 (1984)

15. Kac, V. G., Wakimoto, M.: Modular and conformal invariance constraints in representation theory of affine algebras. Adv. Math. 70 156-236 (1988)

16. Koblitz, N., Rohrlich, D.: Simple factors in the Jacobian of a Fermat curve. Can. J. Math. XXX 1183-1205 (1978)

17. Lemire, F., Patera, J.: Congruence number, a generalization of SU(3) triality. J. Math. Phys. 21 2026-2027 (1980)

18. Lerche, W., Vafa, C., Warner, N.: Chiral rings in $\mathrm{N}=2$ superconformal theories. Nucl. Phys. B324 427-474 (1989)

19. Mathieu, P., Sénéchal, D., Walton, M. A.: Field identification in nonunitary diagonal cosets. Int. J. Mod. Phys. A7 supplement 1B 731-764 (1992)

20. Moore, G., Seiberg, N.: Taming the conformal zoo. Phys. Lett. 220B 422-430 (1989)

21. Ravanini, F.: An infinite class of new conformal field theories with extended algebras. Mod. Phys. Lett. A3 397-412 (1988)

22. Ruelle, Ph., Thiran, E., Weyers, J.: Implications of an arithmetical symmetry of the commutant for modular invariants. Nucl. Phys. B402 693-708 (1993)

23. Schellekens, A. N., Yankielowicz, S.: Field identification fixed points in the coset construction. Nucl. Phys. B334 67-102 (1990)

24. Stanev, Y.: Local extensions of the $\mathrm{SU}(2) \times \mathrm{SU}(2)$ conformal current algebras. Vienna ESI preprint (April 1994)

25. Washington, L. C.: Introduction to Cyclotomic Fields. Springer 1982 
Table. Physical invariants of $s u(3)_{k} \oplus s u(3)_{1} / s u(3)_{k+1}$

\begin{tabular}{|c|c|c|c|}
\hline Level & Physical invariants & & \\
\hline$k \equiv 0(\bmod 3)$ & $\mathcal{A}_{k} \otimes \mathcal{A}_{k+1}$ & $\mathcal{D}_{k} \otimes \mathcal{A}_{k+1}$ & $\mathcal{D}_{k}^{c} \otimes \mathcal{A}_{k+1}$ \\
\hline & $\overline{\mathcal{A}}_{k} \otimes \overline{\mathcal{D}}_{k+1}^{c}$ & $\overline{\mathcal{D}}_{k} \otimes \overline{\mathcal{D}}_{k+1}^{c}$ & $\overline{\mathcal{D}}_{k}^{c} \otimes \overline{\mathcal{D}}_{k+1}^{c}$ \\
\hline$k \equiv 1(\bmod 3)$ & $\overline{\mathcal{A}}_{k} \otimes \overline{\mathcal{A}}_{k+1}$ & $\bar{M}\left(A_{k} A_{k+1}\right)$ & ${ }^{c} \bar{M}\left(A_{k} A_{k+1}\right)^{c}$ \\
\hline & $\overline{\mathcal{D}}_{k}^{c} \otimes \overline{\mathcal{A}}_{k+1}$ & $\overline{\mathcal{A}}_{k} \otimes \overline{\mathcal{D}}_{k+1}^{c}$ & $\overline{\mathcal{D}}_{k}^{c} \otimes \overline{\mathcal{D}}_{k+1}^{c}$ \\
\hline$k \equiv 2(\bmod 3)$ & $\overline{\mathcal{A}}_{k} \otimes \overline{\mathcal{A}}_{k+1}$ & $\overline{\mathcal{A}}_{k} \otimes \overline{\mathcal{D}}_{k+1}$ & $\overline{\mathcal{A}}_{k} \otimes \overline{\mathcal{D}}_{k+1}^{c}$ \\
\hline & $\overline{\mathcal{D}}_{k}^{c} \otimes \overline{\mathcal{A}}_{k+1}$ & $\overline{\mathcal{D}}_{k}^{c} \otimes \overline{\mathcal{D}}_{k+1}$ & $\overline{\mathcal{D}}_{k}^{c} \otimes \overline{\mathcal{D}}_{k+1}^{c}$ \\
\hline$k=4$ & $\overline{\mathcal{A}}_{4} \otimes \overline{\mathcal{E}}_{5}$ & $\overline{\mathcal{D}}_{4}^{c} \otimes \overline{\mathcal{E}}_{5}$ & \\
\hline & $\left(\overline{\mathcal{A}}_{4} \otimes \overline{\mathcal{E}}_{5}\right)\left[\bar{M}\left(A_{4} A_{5}\right)\right]$ & $\left(\overline{\mathcal{A}}_{4} \otimes \overline{\mathcal{E}}_{5}\right)^{c}\left[\bar{M}\left(A_{4} A_{5}\right)\right]^{c}$ & \\
\hline$k=5$ & $\overline{\mathcal{E}}_{5} \otimes \overline{\mathcal{A}}_{6}$ & $\overline{\mathcal{E}}_{5} \otimes \overline{\mathcal{D}}_{6}$ & \\
\hline$k=8$ & $\overline{\mathcal{A}}_{8} \otimes \overline{\mathcal{E}}_{9}(1)$ & $\overline{\mathcal{D}}_{8}^{c} \otimes \overline{\mathcal{E}}_{9}{ }^{(1)}$ & $\overline{\mathcal{A}}_{8} \otimes \overline{\mathcal{E}}_{9}(2)$ \\
\hline & $\overline{\mathcal{D}}_{8}^{c} \otimes \overline{\mathcal{E}}_{9}(2)$ & $\overline{\mathcal{A}}_{8} \otimes \overline{\mathcal{E}}_{9}(2) c$ & $\overline{\mathcal{D}}_{8}^{c} \otimes \overline{\mathcal{E}}_{9}(2) c$ \\
\hline$k=9$ & $\overline{\mathcal{E}}_{9}{ }^{(1)} \otimes \overline{\mathcal{A}}_{10}$ & $\overline{\mathcal{E}}_{9}{ }^{(1)} \otimes \overline{\mathcal{D}}_{10}{ }^{c}$ & $\overline{\mathcal{E}}_{9}{ }^{(2)} \otimes \overline{\mathcal{A}}_{10}$ \\
\hline & $\overline{\mathcal{E}}_{9}{ }^{(2)} \otimes \overline{\mathcal{D}}_{10}{ }^{c}$ & $\overline{\mathcal{E}}_{9}(2) c \otimes \overline{\mathcal{A}}_{10}$ & $\overline{\mathcal{E}}_{9}{ }^{(2) c} \otimes \overline{\mathcal{D}}_{10}{ }^{c}$ \\
\hline$k=20$ & $\overline{\mathcal{A}}_{20} \otimes \overline{\mathcal{E}}_{21}$ & $\overline{\mathcal{D}}_{20}^{c} \otimes \overline{\mathcal{E}}_{21}$ & \\
\hline$k=21$ & $\overline{\mathcal{E}}_{21} \otimes \overline{\mathcal{A}}_{22}$ & $\overline{\mathcal{E}}_{21} \otimes \overline{\mathcal{D}}_{22}{ }^{c}$ & \\
\hline
\end{tabular}

Table. This gives the corresponding WZNW invariant - see Sect.4.1 for the notation, and Sect.2.3 for the correspondence. Thm. 2 says this list is complete. 